\section{GAS HYDRATE AND FREE GAS DETECTION USING SEISMIC QUALITY FACTOR ESTIMATES FROM HIGH-RESOLUTION P-CABLE 3D SEISMIC DATA}

Sunny Singhroha ${ }^{1}$, Stefan Bünz ${ }^{1}$, Andreia Plaza-Faverola ${ }^{1}$, Shyam Chand ${ }^{1,2}$

${ }^{1}$ CAGE - Centre for Arctic Gas Hydrate, Environment and Climate, Department of Geology, UiT The Arctic University of Norway, Tromsø, Norway

${ }^{2}$ Geological Survey of Norway (NGU), Trondheim, Norway

sunny.singhroha@uit.no

stefan.buenz@uit.no

andreia.a.faverola@uit.no

shyam.chand@ngu.no

Original paper date of submission: $20^{\text {th }}$ January, 2015

Revised paper date of submission: $14^{\text {th }}$ August, 2015 
19

20

21

22

23

24

26

27 marine sediments.

\section{ABSTRACT}

Seismic attenuation in gas hydrate and free gas bearing sediments is estimated from high-resolution P-Cable 3D seismic data from Vestnesa Ridge on the Arctic continental margin of Svalbard. P-Cable data have broad bandwidth $(20-300 \mathrm{~Hz})$ which is extremely advantageous in estimating seismic attenuation in a medium. The seismic quality factor $(\mathrm{Q})$, inverse of seismic attenuation, is estimated from the seismic dataset using centroid frequency shift method and spectral ratio method. Centroid frequency shift method establishes a relationship between the change in the centroid frequency of an amplitude spectrum and the $\mathrm{Q}$ value of a medium. Spectral ratio method estimates the $\mathrm{Q}$ value of a medium by studying the differential decay of different frequencies. Broad bandwidth and short offset characteristics of the P-Cable dataset are useful to continuously map Q for different layers throughout the $3 \mathrm{D}$ seismic volume. The centroid frequency shift method is found to be relatively more stable than spectral ratio method. Q values estimated using these two methods are in concordance with each other. The $\mathrm{Q}$ data documents attenuation anomalies in the layers in the gas hydrate stability zone above the BSR and in the free gas zone below. Changes in the attenuation anomalies correlate with small-scale fault systems in the Vestnesa Ridge suggesting a strong structural control on the distribution of free gas and gas hydrates in the region. We argue that high and spatially limited Q anomalies in the layer above the BSR indicate the presence of gas hydrates in marine sediments in this setting. Hence, the presented workflow to analyze Q using high-resolution P-cable 3D seismic data with a large bandwidth can be a potential technique to detect and directly map the distribution of gas hydrates in 
Gas hydrates are crystalline ice like structures normally formed at certain temperature and pressure conditions (Brooks et al., 1986). The temperature and pressure conditions required for gas hydrates formation are available in continental slope and permafrost environments (Sloan, 1998). The presence of marine gas hydrates in continental margins has been confirmed from different drilling activities (Collett and Ladd, 2000; Collett et al., 1999; Riedel et al., 2010; Ryu et al., 2013; Liu et al., 2012; Zhang et al., 2007). Seismic methods are commonly used to remotely identify gas hydrates in the marine sediments. The presence of gas hydrates in the sediments is often indicated in seismic data by a bottom simulating reflection (BSR) (Shipley et al., 1979). It marks a sharp impedance contrast between hydratebearing and gas-charged sediments. The BSR occurs at the base of the hydrate stability zone, which is governed mostly by pressure and temperature conditions (Sloan, 1998). Due to this control, the BSR often mimics the seafloor; therefore, cross-cuts the sedimentary strata (Shipley at al., 1979). Since the presence of gas hydrates increases the velocities, concentration of gas hydrates in sediments is usually estimated using seismic velocity models (Lee and Collett, 2001; Gei and Carcione, 2003; Ecker et al., 1998; Chand et al., 2004). The presence of gas hydrates in sediments has a pronounced effect on amplitude and frequency characteristics of a seismic signal also (Guerin and Goldberg, 2002; Pratt et al., 2003; Chand and Minshull, 2004). Hydrates in sediments show contradicting amplitude characteristics in seismic sections like amplitude blanking (Korenaga et al., 1997) and amplitude enhancements (Nouzé et al., 2004; Yoo et al., 2013, Riedel et al., 2010) at different geological settings. spherical divergence, obliquity factor, scattering etc.) and intrinsic attenuation (due to conversion of vibration energy into heat energy) (Mavko et al., 1998). At seismic 
67 frequencies, analysis on attenuation normally refers to intrinsic attenuation (Mavko et al., 1998) which can be studied through spectral analysis (Jacobson et al., 1981). Since gas hydrate increases the stiffness of the matrix (Jung et al., 2012) and P-wave velocity, it was normally assumed that the sediments saturated with gas hydrates will show lower attenuation (Wood et al., 2000). Unlike P-wave velocity, no unique trend of seismic attenuation in gas hydrates can be observed from the literature; thus making attenuation characteristic of the gas hydrate bearing sediments a debatable topic (Guerin et al., 1999; Wood et al., 2000; Chand et al., 2004; Rossi et al., 2007; Sain et al., 2009; Sain and Singh, 2011; Jaiswal et al., 2012; Dewangan et al., 2014). Laboratory experiments in hydrate bearing sediments indicated increase of attenuation with hydrate saturation (Priest et al., 2006; Best et al., 2013) whereas attenuation estimates from field experiments on gas hydrates indicated contradicting results. For example, studies on well log data (Guerin and Goldberg, 2002; Guerin and Goldberg, 2005; Matsushima, 2005), VSP data (Pratt et al., 2005; Bellefleur et al., 2007) and on crosshole seismic data (Pratt et al., 2003; Bauer et al., 2005) indicated an increase in attenuation. Other studies, mainly on surface seismic data (Dewangan et al., 2014; Rossi et al., 2007; Matsushima, 2006) indicated a decrease in attenuation. The increase (Guerin and Goldberg, 2002; Gei and Carcione, 2003; Chand and Minshull, 2004; Lee and Collet, 2006) and decrease (Dewangan et al., 2014; Sain and Singh, 2011) in attenuation has been explained by using different rock physics models depending on the assumed micro structure of the hydrate and also sediment-hydrate mixtures. Chand and Minshull (2004) suggested that the amount of attenuation not only changes with hydrate saturation but also with the frequency of seismic signal.

The seismic quality factor $(\mathrm{Q})$, inverse of seismic attenuation, can be estimated from the seismic dataset using different methods which includes the amplitude decay method (Badri and Mooney, 1987), the rise time method (Gladwin and Stacey, 1974), the centroid 
92 frequency shift method (Quan and Harris, 1997), wavelet modeling (Jannsen et al., 1985), the pulse broadening method (Hatherly, 1986), the spectral ratio method (Jannsen et al., 1985; Båth, 1982) and the inversion method (Amundsen and Mittet, 1994). Tonn (1991) compared 10 methods of attenuation estimation using VSP seismograms and concluded that no single method is suitable for all situations.

In the present study, we apply two different methods to investigate seismic attenuation in gas hydrate and free gas saturated sediments from Vestnesa Ridge, a deepwater gas hydrate system located offshore west-Svalbard (Figure 1). The quality factor (Q) has been estimated from P-Cable seismic data using the spectral ratio method (Jannsen et al., 1985) and the centroid frequency shift method (Quan and Harris, 1997). The centroid frequency shift method establishes a relationship between the change in the centroid frequency of an amplitude spectrum and the Q value of a medium (Quan and Harris, 1997).

104 On the contrary, the spectral ratio method estimates the Q value of a medium by studying the 105 differential decay of different frequencies (Båth, 1982). Due to limitation of seismic 106 bandwidth in conventional seismic data, it is almost impossible to map Q with high accuracy.

107 Low signal to noise ratio, short bandwidth, source/receiver array directivity and distinct 108 raypaths in a CDP gather are the main problems encountered in Q analysis from conventional surface seismic data (Hustedt and Clark, 1999). But P-Cable surface seismic data is 110 essentially zero-offset (offset varying from 97-143 m) in deep water and has broad bandwidth 111 (20-300 Hz). Raypaths of different traces in a CDP gather of P-Cable data are approximately 112 similar at deep water depth as offset is quite small. Stacked P-Cable data has high signal to 113 noise ratio and the stacking process involves traces with almost similar raypaths. These 114 characteristics of P-cable data match well with the characteristics of VSP data (Galperin, 115 1985) and make P-Cable data suitable for subsurface Q analysis. Moreover, using P-Cable 116 3D seismic data for estimating Q allows us to analyze the spatial distribution of Q which can 
117 be integrated with 3D seismic interpretation. Thereby, we can link Q estimates with 118 anomalies related to the presence of gas hydrate and free gas in the sediments.

\section{STUDY AREA}

120

Our study focuses on the active seeping segment of Vestnesa Ridge, a $\sim 100 \mathrm{~km}$ long

121 gas hydrate charged contourite drift developed over $<20$ Ma oceanic crust offshore west-

122 Svalbard (Figure 1) (Eiken and Hinz, 1993; Vogt et al., 1994, Bünz et al., 2012). The

123 contourite drift is in close proximity to the Molloy and the Knipovich slow-spreading oceanic

124 ridges and it is located between the Molloy and the Spitsbergen Transform Faults (e.g.,

125 Ritzmann et al., 2004). Vestnesa Ridge consists of three main stratigraphic sequences named

126 according to correlation with ODP sites at the Yermak Plateau (YP) (Eiken and Hinz, 1993):

127 the oldest unit (YP1) is of Miocene age and consists dominantly of syn-rift deposits directly

128 lying over the oceanic crust (Eiken and Hinz, 1993; Ritzmann et al., 2004); the middle

129 sequence (YP2) consists of sediments deposited by migrating contour currents; and finally

130 the youngest sequence (YP3), is dominated by margin parallel contour currents and by

131 glacigenic debris flow deposits (Howe et al., 2008).

A gas hydrate system and associated free gas zone exists along Vestnesa Ridge

133 (Hustoft et al., 2009; Petersen et al., 2010; Bünz et al., 2012; Plaza-Faverola et al., 2015).

134 The system is restricted to the upper stratigraphic sequence (YP3) and has a series of gas

135 chimneys and pockmarks associated along the full extent of Vestnesa Ridge. However, only

136 pockmarks located towards the easternmost part of the ridge (where our 3D seismic survey is

137 located; Figure 1) are actively seeping gas at present (Bünz et al., 2012; Smith et al., 2014).

138 Gas chimneys towards the westernmost part of the ridge seem inactive at present but

139 foraminiferal records indicated past activity at around 8000 my ago (Consolaro et al., 2014). 
142 seismic data acquired in 2013 on board R/V Helmer Hanssen (Plaza-Faverola et al., 2015).

143 The system consists of 14 streamers towed parallel behind the ship. The 25 -m-long streamers

144 contain 8 receiver groups each. The streamers are attached to a cross cable towed

145 perpendicular to the vessel's streaming direction and spread by two large trawl doors. The 146 spacing of streamers along the cross cable is $12.5 \mathrm{~m}$. However, due to curvature of the cross 147 cable, the distance between streamers is varying between 6-10 m. The high-resolution P148 Cable system was used together with mini-GI gun $\left(15 / 15 \mathrm{in}^{3}\right)$. The gun was fired at an 149 interval of $6 \mathrm{~s}$ with a firing pressure of 170 bar. Source-receiver offset varies from 97-143 m.

150 Traces have been recorded with $3 \mathrm{~s}$ record length at $0.25 \mathrm{~ms}$ sampling interval.

152 can potentially distort the amplitude spectrum within the main seismic bandwidth. The 153 processing of the high-resolution 3D seismic data mainly included navigational correction, 154 static and tidal correction, binning, bandpass filtering (10-20-300-350), NMO correction and 155 stacking. NMO correction can potentially distort the amplitude spectrum due to NMO stretching. But for short offset seismic data in deep water, this distortion will be too small and 157 can be neglected. 3D Stolt migration was applied using a constant velocity of $1500 \mathrm{~m} / \mathrm{s}$. The 158 spatial resolution of the seismic data is quite high with a bin size of $6.25 \times 6.25 \mathrm{~m}$. The 159 seismic data covers an area of about $14 \mathrm{~km}^{2}$. The data has a broad frequency spectrum 160 ranging from approximately 20 to $300 \mathrm{~Hz}$ (Figure 2a). 


\section{METHODOLOGY}

Amplitude, frequency and phase are three basic attributes of a seismic signal.

164 Quantitative analysis of these attributes is done using different derivations and transforms.

165 For example, quantitative estimation of frequency attribute involves different time-frequency

166 transforms. Reine et al. (2009) discussed the robustness of seismic attenuation measurements

167 using different time-frequency transforms. In the present study, short-time Fourier transform

168 (Gabor, 1946) is used to transform a seismic signal into frequency domain. The data

169 converted to frequency domain is used to study seismic attenuation. We use centroid 170 frequency shift (Quan and Harris, 1997) and spectral ratio method (Jannsen et al., 1985) to

171 estimate Q in gas hydrate and free gas saturated sediments.

\section{Centroid frequency method}

Centroid frequency of an amplitude $\operatorname{spectrum}\left(f_{\mathrm{c}}\right)$ is defined as:

$$
f_{c}=\frac{\sum \mathrm{A}(\mathrm{f}) \mathrm{xf}}{\sum \mathrm{A}(\mathrm{f})}
$$

Where A(f) corresponds to amplitude of frequency (f) in an amplitude spectrum.

Centroid frequency of a signal gives an idea about the energy level of a signal. Since energy of a signal decreases as it propagates in the sub-surface, centroid frequency of an amplitude spectrum shifts towards lower values with further propagation into deeper layers.

179 By plotting the centroid frequency for the entire seismic section, a broad overview about the 180 subsurface seismic attenuation can be established. Quan and Harris (1997) proposed a 181 method to estimate Q using centroid frequency shift analysis. They considered the amplitude spectrum of the received signal $R(f)$ as a function of incident wave $S(f)$ and instrument/medium response $\mathrm{G}(\mathrm{f}) \mathrm{H}(\mathrm{f})$. 

coupling, radiation/transmission coefficients, and phase accumulation effects caused by 187 propagation. $\mathrm{H}(\mathrm{f})$ is a factor which takes into account the effect of intrinsic attenuation on a 188 seismic signal. Since attenuation is proportional to frequency within the seismic bandwidth, 189 response $\mathrm{H}(\mathrm{f})$ can be written as (Johnston et al., 1979):

$$
H(f)=\exp \left(-f \int_{\text {ray }} \alpha_{0} \mathrm{dl}\right)
$$
Where the integral is taken along the ray path, and $\alpha_{0}$ is the attenuation coefficient defined by (Johnston et al., 1979): Where $\mathrm{Q}$ is the quality factor and $\mathrm{v}$ is the velocity of the medium. distribution, Quan and Harris (1997) after rearranging the equations finally came to the 197 following equation:

$$
\int_{\text {ray }} \alpha_{0} \mathrm{dl}=\frac{\mathrm{f}_{\mathrm{s}}-\mathrm{f}_{\mathrm{r}}}{\sigma_{\mathrm{s}}{ }^{2}}
$$

Where $f_{s}$ is the centroid frequency of the source signal (Figure $2 b$ ), $f_{r}$ is the centroid 200 frequency of the received signal, and $\sigma_{\mathrm{s}}^{2}$ is the variance of the source amplitude spectrum.

$$
\sigma_{\mathrm{s}}{ }^{2}=\frac{\int_{0}^{\infty}\left(f-f_{s}\right)^{2} A(f) d f}{\int_{0}^{\infty} A(f) d f}
$$


Where $A(f)$ is the amplitude spectrum of the source signal and other parameters are same as described in the above equation. In order to account for the increase in the variance 204 of amplitude spectrum, $\sigma_{\mathrm{s}}{ }^{2}$, of seismic signal with arrival time, a trend line for $\sigma_{\mathrm{s}}^{2}$ at different 205 arrival times is estimated (Figure 2c). Straight line is fitted to the mean $\sigma_{\mathrm{s}}{ }^{2}$ values.

If velocity and quality factor (Q) is assumed constant in a medium, the final expression for quality factor (Q) can be written (Talukder, 2013) as:

$$
\mathrm{Q}=\frac{\pi \sigma_{\mathrm{s}}^{2} \Delta \mathrm{t}}{\mathrm{f}_{\mathrm{s}}-\mathrm{f}_{\mathrm{r}}}
$$

Where $\Delta \mathrm{t}$ is the total travel time and rest of the parameters are same as described in

210 above equations.

\section{Spectral ratio method} Spectral ratio method is one of the most commonly used methods to estimate Q in a medium. This method takes into account the differential decay of different frequencies.

214 Higher frequencies tend to decay at a much higher rate as compared to lower frequencies 215 while passing through an attenuating medium (Båth, 1982). Differential decay of different 216 frequencies depends upon Q of a medium.

218 estimate $Q$ from seismic data. Amplitude spectrums $\left(A_{1}(\omega)\right.$ and $\left.A_{2}(\omega)\right)$ of two reflections 219 from different depths $\left(Z_{1}\right.$ and $\left.Z_{2}\right)$, can be written as:

$$
A_{1}(\omega)=A_{0}(\omega) G\left(Z_{1}\right) R_{1} e^{-2 \alpha_{1} Z_{1}}
$$

$$
A_{2}(\omega)=A_{0}(\omega) G\left(Z_{2}\right)\left(1-R_{1}{ }^{2}\right) R_{2} e^{-2 \alpha_{1} Z_{1}} e^{-2 \alpha_{2}\left(Z_{2}-Z_{1}\right)}
$$


Where $A_{0}(\omega)$ is the amplitude spectrum of the incident wavelet at $Z=0, G\left(Z_{1}\right)$ and

$223 \mathrm{G}\left(Z_{2}\right)$ accounts for the geometrical spreading and other factors leading to decay in 224 amplitudes, $\mathrm{R}_{1}$ and $\mathrm{R}_{2}$ are reflection coefficients for different boundaries, and $\alpha_{1}$ and $\alpha_{2}$ are 225 the attenuation coefficients. The spectral ratio (SR) of two spectra can be written as:

$$
S R(\omega)=C_{1} e^{-2 \alpha_{2}\left(Z_{2}-Z_{1}\right)}
$$

$$
C_{1}=\frac{G\left(Z_{2}\right)\left(1-R_{1}{ }^{2}\right) R_{2}}{G\left(Z_{1}\right) R_{1}}
$$

Where $C_{1}$ is the ratio of factors related to geometrical spreading and reflection coefficients. Assuming phase velocity $\beta$ to be independent of frequency in spectral ratio (Båth, 1982), natural log of spectral ratio can be written as:

$$
\ln (S R(\omega))=\ln \left(C_{1}\right)-\alpha_{2} \Delta T \beta
$$
where $\Delta \mathrm{T}$ is the time difference between two reflections. Substituting the value of $\alpha$ as $\pi \mathrm{f} /(\mathrm{Q} \beta)$ (Johnston et al., 1979), we get linear relation between $\ln (\mathrm{SR}(\omega))$ and frequency, 234 i.e.,

$$
\ln (S R(\omega))=\ln \left(C_{1}\right)-\left(\frac{\pi \Delta T}{Q}\right) f
$$

237 plot depends on the Q of a medium, and the intercept is related to the geometrical spreading 238 and reflection coefficients which are independent of frequency. Using this concept, Q value can be estimated from the slope of the best fit line in spectral ratio (in logarithmic scale) vs

240 frequency plot. In real data, two wavelets can be picked by windowing two reflections

241 (Figure 3a) and then Fourier transform can be applied to get amplitude spectrum of these two 
242 wavelets (Figure 3b). Spectral ratio method can be applied on these two amplitude spectrums and effective Q of a medium between these reflections can be estimated (Figure 3c).

Analysis using centroid frequency plots has been done to study changes in the 246 centroid frequency with depth. An inline has been selected from seismic data where a BSR is 247 clearly identified by high-seismic amplitudes at about $1.9 \mathrm{~s}$ TWT in the seismic section 248 (Figure 4a) (Bünz et al., 2012; Smith et al., 2014). The BSR separates hydrate-bearing 249 sediments from a $\sim 100 \mathrm{~m}$ thick free gas zone (Hustoft et al., 2009). Other notable features are 250 vertical zones of acoustic transparency or chaotic seismic facies. These are interpreted as 251 vertical fluid-flow features, so-called chimneys. They terminate in seafloor depressions 252 known as pockmarks (Figure 4c) (Bünz et al., 2012).

254 an interval of $5 \mathrm{~ms}$. Enough samples have been taken to ensure that the lowest frequency in 255 the main seismic bandwidth will have at least one wavelength to sample. The derived 256 centroid frequencies are shown in Figure 4b. The centroid frequency decreases significantly 257 beneath the BSR. Prominent low centroid frequency anomalies are observed in the free gas 258 zone especially in the southeastern part of the seismic section (Figure 4b). Gas chimneys are 259 observed in the seismic section. Some gas chimneys also show low centroid frequency 260 anomalies. Some of these anomalies appear to intrude the chimneys from the free gas zone 261 beneath the BSR. Coincidentally, intrusion happens for gas chimneys that have active gas 262 seepage at the seafloor (Figure 4c) (Bünz et al., 2012). 
Variance of an amplitude spectrum $\left(\sigma_{\mathrm{s}}^{2}\right)$ and reference centroid frequency $\left(\mathrm{f}_{\mathrm{s}}\right)$ were calculated to further estimate Q for different layers using centroid frequency shift method. In

267 order to account for an increase in the $\sigma_{\mathrm{s}}{ }^{2}$ with arrival time, $\sigma_{\mathrm{s}}{ }^{2}$ of a seismic signal is plotted 268 with respect to two-way arrival time. The trend line for $\sigma_{\mathrm{s}}{ }^{2}$ at different arrival times is 269 estimated (Figure 2c). The $\sigma_{\mathrm{s}}^{2}$ to be used in equation (7) is calculated from the linear fit parameters of the best fit line. The centroid frequency of a seismic signal at the sea floor is used as a reference centroid frequency for estimating 1-D Q model for every trace. The centroid frequency of the seismic signal in shallow sediments close to the seafloor (shown in

273 Figure 2 b) ranges from $150-185 \mathrm{~Hz}$ for almost all the traces except for few located in a pockmark where it showed lower centroid frequency values. The centroid frequency of the seismic signal at the seafloor is expected to be higher than the centroid frequency of a seismic signal in shallow sediments. After neglecting aberrations, we use $182 \mathrm{~Hz}$ as a reference centroid frequency (Figure 2b). different layers between picked reflections have been estimated (Figure 5a and 6a). Q model derived from one of the traces in the seismic data is shown in Figure 5a-d. Centroid frequencies are calculated at a time interval of $5 \mathrm{~ms}$ for each trace using a sliding time window along the trace (Figure 5a-b). Fluctuations in centroid frequencies make Q estimation

283 difficult. Therefore, in order to reduce these effects, centroid frequencies of traces falling within $31.25 \times 31.25 \mathrm{~m}^{2}$ bin have been stacked to get a centroid frequency trend/curve (Figure 5c). This process also reduces the size of the seismic dataset and makes it computationally convenient. Synthetic centroid frequency curves are generated for different possible Q models (Q varying from 1 to 600 in all the 5 layers). Synthetic centroid frequency 
curves for 5 Q models out of $600^{5}$ possible Q models are shown in green color in Figure $5 \mathrm{~d}$.

These synthetic centroid frequency curves are matched with estimated centroid frequency curves (estimated from traces in $31.25 \times 31.25 \mathrm{~m}^{2}$ bin), and the misfit between the two curves has been computed. The $L_{1}$ norm approach (Claerbout and Muir 1973) has been used to 292 calculate the misfit and to pick the best possible Q model out of the possible range of Q 293 models. The $\mathrm{L}_{1}$ norm approach has been adopted to reduce the significance of the spiky points in the centroid frequency curves. This process of estimating $Q$ has been repeated on all 295 the traces in the seismic data to generate a Q cube.

\section{Observations}

The Q values along one of the inlines (Figure 6a) indicate high $\mathrm{Q}$ anomalies in the layer above the BSR and low Q anomalies below the BSR (Figure 6b). Q slices for different layers give an idea about the lateral variation of Q within a layer. Figure 7 shows $\mathrm{Q}$ slices for different layers illustrating the lateral variation of Q anomalies within a layer. The BSR lies between Q slices in Figure 7c and 7d. We observe particularly low Q values in some areas within the first layer (Figure 7a) coinciding with the location of the chimney structures. Q estimates in the second layer follows a normal trend except for few small patches of high Q the outer rims of the chimney structures (Figure $7 \mathrm{~b}$ ). Just above the BSR, we observe very high Q values, particularly in the southern half of the 3D seismic data (Figure 7c). These anomalies are found to be laterally continuous. On the contrary, Q values in the center part of this slice (Figure 7c) follow the trend of chimneys and Q values are comparatively lower than those in the slice above (Figure 7b). Extremely low Q values have been observed in Q slice corresponding to free gas zone beneath the BSR except for the locations corresponding to that 311 of chimneys (Figure 7d). 


\section{Q estimation} two prominent reflections (Figure 3). We extend this method to estimate Q for the same 4

316 layers between prominent reflections in the seismic data (Figure 6a) as used in centroid 317 frequency shift method. Picked reflections are windowed and spectral ratio method is applied on adjacent reflections to estimate a subsurface Q model. Figure 8 shows different steps involved in the application of the spectral ratio method on one of the traces. The same procedure is repeated on all the traces in the seismic volume to generate a $\mathrm{Q}$ cube.

\section{Observations}

323 above the BSR (Figure 6c). Q estimates based on spectral ratio method in this layer (Figure

324 6c) is comparable to the $\mathrm{Q}$ estimates from centroid frequency shift method (Figure $6 \mathrm{~b}$ ). Q 325 slices for different layers are plotted to further analyze the results (Figure 9). In the plan view, 326 Q estimates vary significantly within the first layer (Figure 9a). In the second layer, small 327 patches of high $\mathrm{Q}$ values coincide with the chimney features similar to that obtained by 328 centroid frequency shift method. Also, we observe high $\mathrm{Q}$ anomalies in the $\mathrm{Q}$ slice 329 corresponding to the layer just above the BSR (Figure 9c). We find that these high Q 330 anomalies are laterally continuous and match well with the anomalies observed in Q slice 331 obtained from centroid frequency shift method (Figure 7c). Beneath the BSR, we observe 332 predominantly low Q values (Figure 9d). However, we also observe some regions with high 333 Q anomalies in the fourth layer roughly corresponding to chimney locations (Figure 9d). 334 Here, results obtained through spectral ratio method (Figure 9d) and centroid frequency shift 
335 method (Figure 7d) do not agree with each other. At the BSR depth, signal strength is significantly reduced and Q estimates from spectral ratio method are extremely unstable. This may be the reason for high Q values observed in some areas below the BSR.

Estimating Q from seismic data is typically accompanied by some uncertainties and

340 limitations. Contribution of reflectivity sequences in calculated amplitude spectrum directly

341 affects Q estimates. In case of thin layers with some periodicity, Earth's reflectivity function

342 contributes in shaping the spectrum of effective recorded signals. Weak reflectivity over a

343 time window (Figure 5a) can also create bias in Q estimates if the noise spectrum is not 344 white. Ning and Wen-kai (2010) discussed in detail about the effect of reflectivity sequences 345 on Q estimates. Spectral ratio method is more sensitive to these effects as Q is estimated from the spectrum of two wavelets. Fluctuations observed in centroid frequency curves is also 347 primarily due to the effect of reflectivity sequences in the recorded signal.

349 frequencies. Different types of scattering can occur depending on the size of the particles 350 which include Rayleigh, Mie and forward scattering (Mavko et al., 1998). Mie scattering is 351 the type of scattering that will occur when the heterogeneity scale length is of the order of the 352 seismic wavelength. The main difference between scattering and intrinsic attenuation is that 353 scattering redistributes wave energy within the medium but does not remove the energy from 354 the overall wavefield whereas intrinsic attenuation converts vibration energy into heat energy 355 (Sato and Fehler, 1997). Intrinsic attenuation quantified using different methods also includes 356 the contribution from scattering attenuation (Spencer et al., 1982). This will directly affect the 357 Q estimates from different methods. 
Processing of seismic data can be another source of error in Q estimation. Ideally, all the processing steps which can potentially alter amplitude spectrum of a seismic signal should be avoided. In P-Cable data, the potential of this problem is significantly reduced as P-Cable data is close to zero offset $(97-143 \mathrm{~m})$, particularly given the water depth in the

362 present study. Frequency distortions due to NMO stretching are negligible for small offsets at 363 deep water depths. Frequency distortions due to Stolt migration are also negligible as layers in the study area are essentially flat. Apart from these two processing steps, no other step has been involved which can potentially influence the analysis. centroid frequency shift method. Picking more reflections and using them as layer boundaries increases the number of layers for which effective Q model will be estimated but decreases the travel time of the layers. Effect of fluctuations in the centroid frequency curve on Q estimates is more pronounced for thinner layers. Thus, accuracy of Q estimates in thinner layers is poorer than thicker layers. Figure 5e shows best fit Q models for different number of

372 layers. When the number of picked layers is increased from 5 to 7 , the instability in the Q estimates can be clearly seen. Therefore, reflections need to be picked properly so that Q can be estimated for different layers with an acceptable accuracy. spectral ratio method (Figure 10d) in layer 3 (which lies just above the BSR) have been 377 plotted to analyze the statistical distribution of Q estimates within a layer. The peak at $Q=600$ observed in the histograms is due to the fact that only Q values up to 600 have been taken into consideration. All Q values greater than 600 will be estimated as 600 and it is extremely difficult to differentiate between different Q values for those higher than approximately 150 .

381 Accuracy of Q estimates decreases for high Q values where it changes very rapidly with 
small change in seismic signal decay. This small amount of decay becomes comparable with the fluctuations caused by other factors which create a problem in Q estimation. Figure $5 \mathrm{~d}$ shows the estimated Q for different layers. From layer 2 to layer 3, estimated Q changes from 160 to 403 , but there is a very small change in tilt of the best fit curve. This limits the accuracy of Q estimates for high Q values and due to this fact, only Q values up to 600 have been taken into consideration (Figure 10).

Given the two methodological approaches for estimating Q, their inherent limitations and the constraints of the 3D seismic, as earlier reported by Quan and Harris (1997) and Matsushima (2006), we also found that the centroid frequency shift method gave more stable Q estimates. Contribution of reflectivity sequences in calculated amplitude spectrum and scattering effects limited the vertical resolution of Q estimates. We tried to do high-resolution Q sampling but accuracy of Q estimates decreased when greater number of layers was used to estimate Q. Reflectivity sequences and scattering effects made the continuous mapping of Q unstable. Q estimates became unreliable for thinner layers. We observed trade-off between the accuracy and resolution.

\section{DISCUSSION}

There are different factors which contribute to intrinsic attenuation of a seismic signal (Toksöz and Johnston, 1981). Major factors which play a crucial role are lithology, fluid type and structural features (Walsh, 1966; Johnston et al., 1979; Toksöz et al., 1979; Toksöz and

401 Johnston, 1981; Winkler et al., 1979; Spencer, 1979; Winkler and Nur, 1982; Murphy et al., details of the sedimentary environment of the study area are not well known but it is believed

404 to be composed of smoothly deposited layers of contourite deposits (Eiken and Hinz, 1993; 405 Howe et al., 2008). Under such geological settings, the major factors which can prominently 
406 change the intrinsic attenuation property of a medium are changes in fluid type and fluid saturation. Changes in gas hydrate saturation within gas hydrate stability zone will sharply change the intrinsic attenuation spatially. Several publications explained the relationship between seismic attenuation and fluid saturation (O'Connell and Budiansky, 1977; Mavko and Nur, 1979; Spencer, 1979; Murphy et al., 1986; O’Hara, 1989; Pointer et al., 2000;

411 Prasad and Nur, 2003; Rapoport et al. 2004). In addition, structural features scatter the seismic signal and contribute significantly to the estimated intrinsic attenuation (Hamilton and Mooney, 1990). It is thus challenging to distinguish between scattering attenuation and intrinsic attenuation (Wennerberg, 1993). The Q parameter estimated for quantifying intrinsic attenuation of a medium also includes the effects from scattering attenuation (Spencer et al., 1982). Possible effects of gas hydrates and free gas on Q estimates is studied by estimating Q values for different layers in the gas hydrate stability zone and free gas zone. The spatial analysis of the Q estimates from the 3D seismic data then allows us to recognize structures and areas that can be related to the presence of gas hydrates in marine sediments even in the absence of seismic velocity control. method and spectral ratio method. Q values estimated in deeper layers (L2, L3, and L4) using 423 these two methods are found to be in concordance with each other and Q values in layers just 424 above the BSR (L2 and L3) are in good agreement with the Q values normally observed in 425 the gas hydrate bearing marine sediments (Wood et al., 2000). Q estimates in the first layer 426 (L1) do not correspond well. Noisy amplitude spectrum near the sea floor (Dewangan et al. 427 2014) and fluctuating spectral ratio (Figure 8c) can be the possible reason for the unstable Q 428 estimates from spectral ratio method in the first layer. However, in the context of this 429 analysis, it is important to study relative changes in Q particularly along Q slices throughout 
430 the whole volume as these might be related to the type of pore fluid and saturation in a given area or structure.

433 7c and Figure 9c). Below the BSR, the centroid frequency (Figure 4b) and Q values of both 434 methods drop significantly (Figure 7d and Figure 9d). Very low Q values are observed below 435 the BSR except for the locations below chimneys, where high Q is observed (Figure 7d and 436 Figure 9d). High free gas concentration can be the reason for rapid attenuation of the seismic 437 signal below the BSR. The strength of the BSR in the seismic data (Figure 4a) also gives 438 some indication about the accumulation of free gas in the region which is estimated to be as 439 high as $1.5-2 \%$ of pore space (Hustoft et al., 2009). In gas chimneys, seismic signal 440 significantly attenuates due to scattering especially in shallow seafloor features like 441 pockmarks. Low signal strength accompanied with seismic blanking in the gas chimneys 442 make Q estimates in gas chimneys unreliable especially at deeper depths.

444 be stated that the background Q values in the marine sediments at the BSR depth is in the 445 range of 60-90. If $\mathrm{Q}$ values in the layer L3 (layer above the BSR) above potentially gas saturated sediments $(\mathrm{Q}<30$ below the BSR in the layer L4) are selectively picked (Figure 10b and 10e) and compared with the overall distribution of Q values in the layer (Figure 10c and 448 10f), relatively higher Q values have been observed above potentially gas saturated sediments 449 (Figure 10). Particularly the variable distribution of extended zones of high Q mapped on Q 450 slices of the 3D data (Figure 11b), in comparison to adjacent areas with lower Q, points towards variable pore fluid type and/or saturation in this strata. There is no indication from

452 the seismic data to expect significant lithologic changes in this rather homogeneous 453 sedimentary environment. Therefore, we attribute this effect to the presence of gas hydrates 
454 in the sediments and suggest that gas hydrate saturated sediments exhibit high Q values 455 within the frequency range used in the study. This observation is supported by the fact that both Q analysis methods match well in the distribution of Q above the BSR. In contrast, areas with very low Q below the BSR indicate the presence of free gas (Figure 11c).

It is difficult to estimate accurate $\mathrm{Q}$ for high $\mathrm{Q}$ value areas as discussed earlier. Therefore it becomes difficult to state exact Q value in gas hydrate saturated sediments. But from the statistical analysis of the results obtained from both methods (Figure 10), it can be stated that high Q values are observed in gas hydrate saturated sediments. Earlier studies on seismic attenuation conducted in the nearby locations also indicated elevated Q values above 463 the BSR (Rossi et al., 2007). Hence, we argue that Q analysis of high-resolution P-Cable 3D 464 seismic data with a large bandwidth can detect and outline spatially limited areas of gas 465 hydrate occurrence in marine sediments.

467 profile to derive a velocity model. This profile lies approximately $10 \mathrm{Km}$ southward to our 468 study area (shown in Figure 1) and can be used to interpret the results of the Q analysis. High 469 gas concentrations exist beneath the BSR towards the southwestern half of the Vestnesa 470 Ridge (Hustoft et al., 2009). Similarly, low Q values beneath the BSR in the southwestern 471 half of the 3D seismic data may indicate the presence of elevated gas concentrations at this 472 location (Figures $7 d$ and 9d). The gas chimneys that align at the crest of the Vestnesa Ridge 473 separate this southwestern half from the northeastern half where Q values are generally 474 higher. A similar behavior is observed above the BSR where high Q values in the 475 southwestern half may indicate higher concentrations of gas hydrates than in the northeastern 476 half. Hustoft et al. (2009) and Bünz et al. (2012) showed that the fluid flow system in the 477 Vestnesa Ridge is topographically controlled and that gas migrates to the crest of the ridge 
478 beneath the BSR, mostly from the southwestern half. Hence, gas availability may be higher in 479 the southwestern half.

In a more recent study, Plaza-Faverola et al. (2015) showed that small-scale fault systems exist at the crest of the Vestnesa Ridge documenting a tectonic control of gas 482 leakage. Fault systems mapped by Plaza-Faverola et al. (2015) at approximately the BSR 483 depth coincide strikingly with the boundaries of abrupt Q changes within layer 3 and 4 above and beneath the BSR, respectively (Figure 11a-c). Changes in Q within a layer are attributed with a variable pore fluid fill. Hence, the Q analysis indicates that fluid distribution in the region is strongly controlled by fault systems in the Vestnesa Ridge. Fault 1 in Figure 11a delimits the southwestern part indicating higher fluid concentrations in both hydrates above the BSR and free gas below. This area also includes the two most active chimneys on the Vestnesa Ridge (Figure 4c) (Bünz et al., 2012; Smith et al., 2014) corroborating our results that gas is more readily available at this location to either leak to the seafloor or to be bound into gas hydrates. As gas migrate upslope in north-east direction (Figure 11d; Hustoft et al., 2009), they are trapped by the regional fault 1 and utilize the fault plane as migration pathway into the free gas zone beneath the BSR.

Q values between fault 1 and 2 indicate lower concentrations of gas hydrates (Figure 11b) and free gas (Figure 11c). Coincidentally, several of the chimneys located in this fault block are inactive. The reduced availability of free gas in this fault block might explain this 497 observation or that most gas has vented through the chimneys. Also other areas of the 3D seismic volume clearly indicate a relationship between Q values and the mapped fault system, e.g. to the northeast of fault 2 or between fault 2 and fault 4 (Figure 11a-c). Together, these 500 results suggest that the availability of free gas is one of the major factors in the accumulation 501 of gas beneath the BSR and the formation of gas hydrates above it, and that the availability of 
502 free gas clearly seems to be controlled by the structural setting supporting the findings of 503 Plaza-Faverola et al. (2015).

All the Q slices clearly exhibit the vertical fluid flow features in this area (Figures 7 and 9). However, there are still some interesting subtleties that can be noted from the Q data.

506 When centroid frequencies are plotted for a seismic section, they didn't drop rapidly at some 507 places in the northwestern and central parts (Figure 4b) of the seismic section. It is possible 508 that these frequency anomalies might be related to variable concentrations of gas within the 509 free gas zone beneath the BSR. Lower amounts of free gas might indicate that the fluid flow 510 features like gas chimneys in nearby locations may lack a gas source. Bünz et al. (2012) 511 documented acoustic flares in the water column and shallow high amplitudes in upper $50 \mathrm{~m}$ 512 of these fluid flow features. In their study they show that the chimneys in the central part of 513 the 3D seismic volume and some chimneys in northwestern part are inactive as compared to 514 chimneys in the southeastern part of the volume (Figure 4c). On the contrary, the active 515 chimneys documented by Bünz et al. (2012) show low frequency anomalies in the lower part 516 of the chimney just above the BSR (Figure 4b). It might indicate an active migration of gas 517 from the free gas zone into the chimney structures supplying the seafloor seep with gas. Low centroid frequencies have been observed in regions where pockmark features

519 have been observed. This can be due to prominent scattering at pockmarks or attenuation of 520 the seismic energy within 5-10 m of sediments below the sea floor possibly resulting from the 521 presence of hydrates and/or carbonates. Prominent scattering in pockmarks and within 522 chimneys significantly reduced the signal strength and made it difficult to image Q in gas 523 chimneys at deeper depth. Low signal strength and seismic blanking in gas chimneys reduces 524 the accuracy of Q estimates in gas chimneys. But still Q values with limited accuracy have 525 been used to study gas chimneys. Both Q estimation methods show small patches of high Q 
526 values associated with chimney features at medium depth beneath seafloor and BSR (Figure

$5277 \mathrm{~b}$ and Figure 9b). However, the centroid frequency shift method depicts high $\mathrm{Q}$ at the rim of

528 the chimneys possibly indicating that chimneys are lined with hydrates, an interesting though

529 speculative suggestion, although it would fit with theoretical models for chimneys structures

530 (Liu and Flemings, 2007).

\section{SUMMARY}

532

We applied the centroid frequency shift method and spectral ratio method to study

533 seismic attenuation in gas hydrate and free gas saturated sediments using high-resolution P-

534 Cable 3D seismic data from Vestnesa Ridge on the Arctic continental margin of Svalbard.

535 We estimated Q values for different layers to develop a subsurface 3D Q model. We observed

536 high $\mathrm{Q}$ values above the prominent $\mathrm{BSR}$ and low $\mathrm{Q}$ values $(\mathrm{Q} \approx 10-30)$ below the $\mathrm{BSR}$.

537 Anomalies observed in Q slices obtained from two different methods are found in

538 concordance with each other. But we got relatively more stable Q values from centroid

539 frequency shift method.

After performing a statistical analysis, we found that an increase in Q values in

541 certain, spatially limited areas above the BSR can probably be associated with the presence of

542 gas hydrates. Under this premise, Q analysis of high-resolution P-Cable 3D seismic data is

543 thus an effective method for the detection and mapping of gas hydrate occurrences in marine

544 sediments. Q values estimated for the strata below the BSR are very low as a consequence of

545 the occurrence of gas trapped in the free gas zone beneath hydrate-bearing strata.

546 Faults that exist throughout the Vestnesa Ridge coincide with the Q anomalies in the 547 layers above and below the BSR corroborating recent findings and directly showing that the 548 structural setting and tectonic activity in the region control the availability and spatial 
549 distribution of free gas and gas hydrates in the Vestnesa Ridge. The availability of gas in

550 certain spatially limited areas also might explain the present seepage from some of the

551 chimneys on the Vestnesa Ridge whereas other chimneys are dormant. Low seismic signal

552 strength accompanied by amplitude blanking makes it difficult to accurately image Q in gas

553 chimneys. But still with limited accuracy, we observed high Q values in gas chimneys in Q

554 slices hinting towards the presence of gas hydrates in gas chimneys. of Excellence funding scheme, project number 223259. We thank the crew of R/V Helmer

558 Hanssen and those who contributed to P-Cable data acquisition. We are also thankful to Ingo 559 Pecher (Associate Editor), Nathan Bangs, Giuliana Rossi, and Kalachand Sain for their 560 constructive comments. zero-offset, vertical seismic profile data: Geophysics, 59, 500- 517. waves in unconsolidated sediments: Geophysics, 52, 772- 784. Båth, M., 1982, Spectral Analysis in Geophysics: Elsevier Science.

568 Ray based cross-well tomography for P-wave velocity, anisotropy, and attenuation structure 569 around the JAPEX/JNOC/GSC et al. Mallik 5L-38 gas hydrate production research well, in S. 570 R. Dallimore, and T. S. Collet, eds., Scientific results from the Mallik 2002 Gas Hydrate 
571 Production Research Well Program, Mackenzie Delta, Northwest Territories, Canada:

572 Geological Survey of Canada, Bulletin 585, 1-21.

Behura, J., 2009, Estimation and Analysis of Attenuation Anisotropy: Doctoral thesis,

574 Colorado School of Mines.

575

Bellefleur, G., M. Riedel, T. Brent, F. Wright, and S. R. Dallimore, 2007, Implication

576

of seismic attenuation for gas hydrate resource characterization, Mallik, Mackenzie Delta,

577 Canada: Journal of Geophysical Research, 112, B10311, doi:10.1029/2007JB004976.

Best, A. I., J. A. Priest, C. R. I. Clayton, and E. V. L. Rees, 2013, The effect of

methane hydrate morphology and water saturation on seismic wave attenuation in sand under shallow sub-seafloor conditions: Earth and Planetary Science Letters, 368, 78-87.

McDonald, 1986, Association of gas hydrates and oil seepage in the Gulf of Mexico: Organic

Geochemistry, 10, 221-234, doi: 10.1016/0146-6380(86)90025-2. attenuation: a case study for Malik 2L-38 well data, Mackenzie delta, Canada: Geophysical Research Letters, 31, L14609, doi:10.1029/2004GL020292. 
Claerbout, J. F., and F. Muir, 1973, Robust modeling with erratic data: Geophysics, 38, 826-844.

595

Collett, T. S., and J. Ladd, 2000, Detection of gas hydrate with downhole logs and assessment of gas hydrate concentrations (saturations) and gas volumes on the Blake Ridge with electrical resistivity log data, in C. K. Paull, R. Matsumoto, P. J. Wallace, and W. P. 598 Dillon, eds., Proceedings of the Ocean Drilling Program, Scientific results: Texas A \& M 599 University, College station Texas, 164, 179-191. 1999, Detailed evaluation of gas hydrate reservoir properties using JAPEX/JNOC/GSC Mallik well 2L-38 gas hydrate research well downhole well-log displays, in S. R. Dallimore, T. Uchida, and T. S. Collett, eds., Scientific Results from JAPEX7JNOC/GSC Mallik 2L-38 604 gas hydrate well, Mackenzie Delta, North west Territories, Canada, Geological Survey of 605 Canada Bulletin 544, 295-312. 2014, Carbon isotope $\left(\delta^{13} \mathrm{C}\right)$ excursions suggest times of major methane release during the last $14 \mathrm{ka}$ in Fram Strait, the deep-water gateway to the Arctic: Climate of the Past Discussions, 10, 4191-4227, doi:10.5194/cpd-10-4191-2014. of seismic attenuation of gas hydrate bearing sediments from multi-channel seismic data: A case study from Krishna-Godavari offshore basin: Marine and Petroleum Geology, 58, Part A, 356-367, doi:10.1016/j.marpetgeo.2014.05.015. structure from seismic AVO: Geophysics, 63, 1659-1669. 
Eiken, O., and K. Hinz, 1993, Contourites in the Fram Strait: Sedimentary Geology,

$61782,15-32$.

618

Gabor, D., 1946, Theory of communication: Journal of the Institution of Electrical

619 Engineers, 93, no. 26, 429-457.

620

Galperin, E. I., 1985, Vertical seismic profiling and its exploration potential: Springer,

621 doi: 10.1007/978-94-009-5195-2.

622 Gei, D., and J. M. Carcione, 2003, Acoustic properties of sediments saturated with gas

623 hydrate, free gas and water: Geophysical prospecting, 51, 141-157.

624 Gladwin, M. T., and F. D. Stacey, 1974, Anelastic degradation of acoustic pulses in 625 rocks: Physics of the Earth and Planetary Interiors, 8, 332- 336.

626 Guerin, G., and D. Goldberg, 2002, Sonic waveform attenuation in gas hydrate627 bearing sediments from the Mallik 2L-38 research well, Mackenzie Delta, Canada: Journal of 628 Geophysical Research, 107, no. B5, 2088, doi:10.1029/2001JB000556.

629 Guerin, G., and D. Goldberg, 2005, Modeling of acoustic wave dissipation in gas 630 hydrate-bearing sediments: Geochemistry, Geophysics, Geosystems, 6, Q07010, 631 doi:10.1029/2005GC000918. 
Hamilton, R. M., and W. D. Mooney, 1990, Seismic-wave attenuation associated with crustal faults in the new madrid seismic zone: Science, 248, no. 4953, 351-354, doi: $10.1126 /$ science.248.4953.351.

638 Hatherly, P. J., 1986, Attenuation measurements on shallow seismic refraction data:

639 Geophysics, 51, 250- 254. seismic attenuation measurements: Geophysical Prospecting, 47, 1105-1119. methane-venting province in sediments on $<20$ Ma young oceanic crust in the Fram Strait, 646 offshore NW-Svalbard: Earth and Planetary Science Letters, 284, no. 1-2, 12-24. wave data- Part II: Attenuation versus depth using spectral ratios: Geophysics, 46, 152-162.

650 of hydrates in faulted, fine-grained sediments of Krishna-Godavari Basin: full waveform 651 inversion: Journal of Geophysical Research, 117, B10305, doi:10.1029/2012JB009201. shallow marine sediments from vertical reflection seismograms: Geophysical Prospecting, 
Johnston, D. H., M. N. Toksöz, and A. Timur, 1979, Attenuation of seismic waves in dry and saturated rocks: II. Mechanisms: Geophysics, 44, 691-711.

658 bearing sands: Numerical study using discrete element method simulations: Journal of

659 Geophysical Research, 117, B04202, doi: 10.1029/2011JB009040.

660 Korenaga, J., W. S. Holbrook, S. C. Singh, and T. A. Minshull, 1997, Natural gas 661 hydrates on the southeast US margins: Constraints from full waveform and traveltime 662 inversions of wide angle seismic data: Journal of Geophysical Research, 102, 15345-15365. sediments: Geophysics, 66, 763-771.

666 form velocity logs on Hydrate ridge, offshore Oregon, USA, in A. M. Tréhu, G. Bohrmann, 667 M. E. Torres, and F. S. Colwell, eds., Proceedings of the Ocean Drilling Program, Scientific 668 Results, 204: College Station TX (Ocean Drilling Program), 1-25. 669 doi:10.2973/odp.proc.sr.204.103.2006.

671 Experimental simulation of the exploitation of natural gas hydrate: Energies, 5, 466-493, doi: $67210.3390 /$ en5020466. 
Matsushima, J., 2005, Attenuation measurements from sonic waveform logs in

677

678

679

680

681

682

683

684

685

686

687

688

689

690

691

692

693

694

695

696

697

methane hydrate-bearing sediments at the Nankai Trough exploratory well off Tokai, central Japan: Geophysical Research Letters, 32, L03306, 1-5, doi: 10.1029/2004GL021786.

Matsushima, J., 2006, Seismic wave attenuation in methane hydrate-bearing sediments: vertical seismic profiling data from the Nankai Trough exploratory well, offshore Tokai, central Japan: Journal of Geophysical Research, 111, B10101, doi:10.1029/2005JB004031.

Mavko, G., T. Mukerji, and J. Dvorkin, 1998, The Rock Physics Handbook -Tools for Seismic Analysis in Porous Media: Cambridge University Press.

Mavko, G. M., and A. Nur, 1979, Wave attenuation in partially saturated rocks: Geophysics, 44, 161-178.

Murphy, W. F., K. W. Winkler, and R. L. Kleinberg, 1986, Acoustic relaxation in sedimentary rocks: Dependence on grain contacts and fluid saturation: Geophysics, 51, 757766.

Ning, T., and L. Wen-kai, 2010, Improve Q estimates with spectrum correction based on seismic wavelet estimation: Applied Geophysics, 7, 217-228, DOI: 10.1007/s11770-0100252-2.

Nouzé, H., P. Henry, M. Noble, V. Martin, and G. Pascal, 2004, Large gas hydrate accumulations on the eastern Nankai Trough inferred from new high-resolution 2-D seismic data: Geophysical Research Letters, 31, L13308, doi:10.1029/2004GL019848. cracked solids: Journal of Geophysical Research, 82, no. 36, 5719-5735. 
O’Hara, S. G., 1989, Elastic-wave attenuation in fluid-saturated Berea sandstone: zones at the Buena Vista Hills reservoir, California: Geophysics, 67, 1061-1070. resolution P-Cable 3D seismic imaging of gas chimney structures in gas hydrated sediments 704 of an Arctic sediment drift: Marine and Petroleum Geology, 27, no. 9, 1981-1994, doi: 705 10.1016/j.marpetgeo.2010.06.006. Technology: P-Cable High-Resolution Seismic: Oceanography, 22, no. 1, 85.

709 Franek, 2015, Role of tectonic stress in seepage evolution along the gas hydrate charged 710 Vestnesa Ridge, Fram Strait: Geophysical Research Letters, 42, no. 3, 733-742, doi: $71110.1002 / 2014 G L 062474$. 
Pratt, R. G, F. Hou, K. Bauer, and M. Weber, 2005, Waveform tomography images of

720 velocity and inelastic attenuation from the Mallik 2002 crosshole seismic surveys, in S. R.

721 Dallimore, and T. S. Collet, eds., Scientific results from the Mallik 2002 Gas Hydrate

722 Production Research Well Program, Mackenzie Delta, Northwest Territories, Canada:

723 Geological Survey of Canada, Bulletin 585, 1-14.

724

Priest, J. A., A. I. Best, and C. R. I. Clayton, 2006, Attenuation of seismic waves in 725 methane gas hydrate-bearing sand: Geophysical Journal International, 164, 149-159, doi: 726 10.1111/j.1365-246X.2005.02831.x.

Quan, Y., and J. M. Harris, 1997, Seismic attenuation tomography using the 728 frequency shift method: Geophysics, 62, 895-905. gas fields based on seismic inelasticity effect: The Leading Edge, 23, 276-278. measurements using fixed- and variable-window time-frequency transforms: Geophysics, 74, no. 2, WA123-WA135, doi: 10.1190/1.3043726. of a fractured gas hydrate system in the Krishna-Godavari basin offshore India: Marine and 736 Petroleum Geology, 27, 1476-1493, doi: 10.1016/j.marpetgeo.2010.06.002. ocean transition: A sheared margin study: Geophysical Journal International, 157, 683-702. 

tomography: an application to gas-hydrate and free-gas detection: Geophysical Prospecting, 55, 655-669.

744 Kim, and D. G. Yoo, 2013, Scientific results of the second gas hydrate drilling expedition in 745 the Ulleung basin (UBGH2): Marine and Petroleum Geology, 47, 1-20, doi: 746 10.1016/j.marpetgeo.2013.07.007. 748 reflector in the Makran Accretionary Prism, Arabian Sea: Marine and Petroleum Geology, 749 28, no. $10,1838-1843$. 751 observations for gas-hydrate-bearing sediments on the western margin of India: Marine 752 Geophysical Researches, 30, no. 3, 137-145, DOI: 10.1007/s11001-009-9073-1. and J. L. Worzel, 1979, Seismic evidence for widespread possible gas hydrate horizons on

757 Continental slopes and Rises: The American Association of Petroleum Geologists Bulletin, $758 \quad 63,2204-2213$. 
762 injection via bubble transport into the upper Arctic Ocean from the hydrate - charged

763 Vestnesa Ridge, Svalbard: Geochemistry, Geophysics, Geosystems, 15, 1945-1959, 764 doi:10.1002/2013GC005179. of pore fluids: Journal of Geophysical Research, 84, 7521-7523.

768 dissipation: Geophysics, 47, 16-24, doi: 10.1190/1.1441275. ratio method vs centroid frequency shift method: CSPG CSEG CWLS GeoConvention 2013, 771 Integration: Geoscience Engineering Partnership, Calgary, Canada.

773 reprint series no. 2, Society of Exploration Geophysicists. 
Walsh, J. B., 1966, Seismic Wave Attenuation in Rock due to Friction: Journal of 782

Geophysical research, 71, 2591-2599.

783

Wennerberg, L., 1993, Multiple-scattering Interpretations of coda-Q Measurements:

784 Bulletin of the Seismological Society of America, 83, 279-290.

785

Winkler, K. W., and A. Nur, 1982, Seismic attenuation: Effects of pore fluids and 786 frictional-sliding: Geophysics, 47, 1-15.

Winkler, K., A. Nur, and M. Gladwin, 1979, Friction and seismic attenuation in rocks:

788 Nature, 277, 528-531.

789

Wood, W. T., W. S. Holbrook, and H. Hoskins, 2000, In situ measurements of P-wave 790 attenuation in methane hydrate and gas bearing sediments on the Blake Ridge, in C. K., Paull, 791 R. Matsumoto, P. J. Wallace, and W. P. Dillon, eds., Proceedings of the Ocean Drilling 792 Program, Scientific Results, 164, 265-272.

794 Riedel, 2013, Occurrence and seismic characteristics of gas hydrate in the Ulleung basin, East 795 Sea offshore Korea: Marine and Petroleum Geology, 47, 236-247, 796 doi:10.1016/j.marpetgeo.2013.07.001.

798 Humphrey, and GMS-1 Science Team, 2007, Successful and surprising results for China's 799 first gas hydrate drilling expedition: Fire in the Ice, 7, no. 3, 6-9. 


\section{LIST OF FIGURES}

802

Figure 1 Bathymetry map showing the location of our study area at Vestnesa Ridge,

803 on the west-Svalbard continental margin. The inset figure shows a seafloor time-structure map derived from the 3D seismic data. Key inlines and crosslines are indicated on this seafloor map. Two small boxes (B1 and B2) show the location of centroid frequency curves plotted in Figure 5. A velocity model was derived from the multi-channel seismic (MCS) line by Hustoft et al. (2009) (see also Figure 11d).

Figure 2 a) Amplitude spectrum of the seismic signal near the seafloor. b) Centroid 809 frequency of seismic signal in shallow sediments near the seafloor for different traces in 810 seismic data. The black line shows the assumed centroid frequency at the seafloor. c) The 811 blue curve shows the mean variance of the amplitude spectrum $\left(\sigma_{\mathrm{s}}{ }^{2}\right)$ at different arrival times.

812 The red line shows the best fit line for changes of mean variance with two way travel time.

Figure 3 a) Sea floor reflection and BSR picked from a trace located at $26^{\text {th }}$ inline and $814260^{\text {th }}$ crossline (see Figure 1 for location). b) Amplitude spectrum of the picked wavelets. c) 815 Plot of spectral ratio (in logarithmic scale) vs frequency. The red curve shows the best fit line 816 ( $\mathrm{L}_{1}$ norm) in this plot. Q is derived from the slope of the best fit line.

Figure 4 a) Selected seismic section (Inline 115, see Figure 1 for location) from the

818 3D seismic data. b) Centroid frequency plot corresponding to the seismic section. Black 819 arrows indicate the possible sub-surface fluid migration through gas chimneys and 820 subsequent seafloor gas seepage. c) Acoustic flares documenting active seepage (modified 821 from Bünz et al., 2012). The black line shows the location of the inline shown in a) and b). 
824 L2, L3, L4 and L5 intervals. b) Centroid frequencies calculated in the $5 \mathrm{~ms}$ sliding time 825 window (Figure 5a). c) Centroid frequency trend/curve for 25 traces in a $31.25 \times 31.25 \mathrm{~m}^{2}$ 826 small box B1 (Figure 1). d) The black curve shows the centroid frequency curve. The green 827 curves show the synthetic centroid frequency curves corresponding to Q model $1\left(\mathrm{Q}_{\mathrm{L} 1}=500\right.$, $828 \mathrm{Q}_{\mathrm{L} 2}=400, \mathrm{Q}_{\mathrm{L} 3}=300, \mathrm{Q}_{\mathrm{L} 4}=200$, and $\left.\mathrm{Q}_{\mathrm{L} 5}=100\right), \mathrm{Q}$ model $2\left(\mathrm{Q}_{\mathrm{L} 1}=70, \mathrm{Q}_{\mathrm{L} 2}=100, \mathrm{Q}_{\mathrm{L} 3}=200\right.$, $829 \mathrm{Q}_{\mathrm{L} 4}=300$, and $\left.\mathrm{Q}_{\mathrm{L} 5}=500\right), \mathrm{Q}$ model $3\left(\mathrm{Q}_{\mathrm{L} 1}=150, \mathrm{Q}_{\mathrm{L} 2}=125, \mathrm{Q}_{\mathrm{L} 3}=100, \mathrm{Q}_{\mathrm{L} 4}=75\right.$, and $\left.\mathrm{Q}_{\mathrm{L} 5}=50\right), \mathrm{Q}$ 830 model $4\left(\mathrm{Q}_{\mathrm{L} 1}=50, \mathrm{Q}_{\mathrm{L} 2}=75, \mathrm{Q}_{\mathrm{L} 3}=150, \mathrm{Q}_{\mathrm{L} 4}=30\right.$, and $\left.\mathrm{Q}_{\mathrm{L} 5}=200\right)$, and $\mathrm{Q}$ model $5\left(\mathrm{Q}_{\mathrm{L} 1}=90\right.$, $831 \mathrm{Q}_{\mathrm{L} 2}=65, \mathrm{Q}_{\mathrm{L} 3}=50, \mathrm{Q}_{\mathrm{L} 4}=40$, and $\mathrm{Q}_{\mathrm{L} 5}=30$ ). The red curve shows the synthetic centroid frequency 832 curve for the best fit ( $\mathrm{L}_{1}$ norm) Q model. b) 5 layer best fit Q model (red) and 7 layer best fit 833 Q model (green) for a centroid frequency curve (location shown by a small box B2 in Figure $8341)$.

Figure 6 a) Seismic section of Inline 69 (see Figure 1 for location) with picked seafloor and three major subsurface reflections. L1, L2, L3, and L4 show the layers for which

837 Q is estimated. b) Overlay of seismic section and Q estimates obtained from centroid 838 frequency shift method. c) Overlay of seismic section and Q estimates obtained from spectral 839 ratio method. Figure 7 Q slices for different layers estimated using centroid frequency shift method.

841 Q slices (a), (b), (c), and (d) correspond to layers L1, L2, L3, and L4 (Figure 6a) respectively. Figure 8 a) Seismic trace corresponding to $98^{\text {th }}$ inline and $686^{\text {th }}$ crossline (see Figure 1 843 for location). L1, L2, L3, and L4 are four layers between five reflections $\left(\mathrm{R}_{1}, \mathrm{R}_{2}, \mathrm{R}_{3}, \mathrm{R}_{4}\right.$, and $844 \mathrm{R}_{5}$ ). b) $\mathrm{AS}_{1}, \mathrm{AS}_{2}, \mathrm{AS}_{3}, \mathrm{AS}_{4}$, and $\mathrm{AS}_{5}$ are amplitude spectra calculated over time windows $\mathrm{R}_{1}$, $845 R_{2}, R_{3}, R_{4}$, and $R_{5}$ respectively. c), d), e), and f) show spectral ratio vs frequency plot. The red 846 lines show the best fit line derived using $\mathrm{L}_{1}$ norm. $\mathrm{Q}_{\mathrm{L} 1}, \mathrm{Q}_{\mathrm{L} 2}, \mathrm{Q}_{\mathrm{L} 3}$, and $\mathrm{Q}_{\mathrm{L} 4}$ are the derived Q 847 values for layers L1, L2, L3, and L4 respectively. 
Figure 9 Q slices for different layers estimated using spectral ratio method. Q slices

849 (a), (b), (c), and (d) correspond to layers L1, L2, L3, and L4 (Figure 6a) respectively.

851 the layer L3. b) Histogram of Q values obtained using centroid frequency shift method in the 852 layer L3 with $\mathrm{Q}<30$ (high concentration of free gas) in the layer L4. c) Ratio of histogram $b$ 853 and histogram a. d) Histogram of Q values obtained using spectral ratio method in the layer 854 L3. e) Histogram of Q values obtained using spectral ratio method in the layer L3 with Q $<30$ 855 (high concentration of free gas) in the layer L4. f) Ratio of histogram e and histogram d. 856 Layers L1, L2, L3 and L4 are shown in Figure 6a.

Figure 11 a) Variance map obtained from a time slice at BSR depth showing several 858 faults (Plaza-Faverola et al., 2015) and gas chimneys (Bünz et al., 2012) piercing through the 859 subsurface. b) Q slice obtained by overlaying (through 50\% transparency) Q slices from 860 spectral ratio method (Figure 9c) and centroid frequency shift method (Figure 7c) in the layer 861 L3 (layer above the BSR). c) Q slice obtained by overlaying (through $50 \%$ transparency) Q 862 slices from spectral ratio method (Figure 9d) and centroid frequency shift method (Figure 7d) 863 in the layer L4 (layer below the BSR). d) P-wave velocity cross-section derived using multi864 channel seismic profile (see Figure 1 for location) across Vestnesa Ridge (modified from 865 Hustoft et al., 2009). Arrows in the Figure show upslope gas migration and its leakage from 866 the Vestnesa Ridge. 
Figure 1

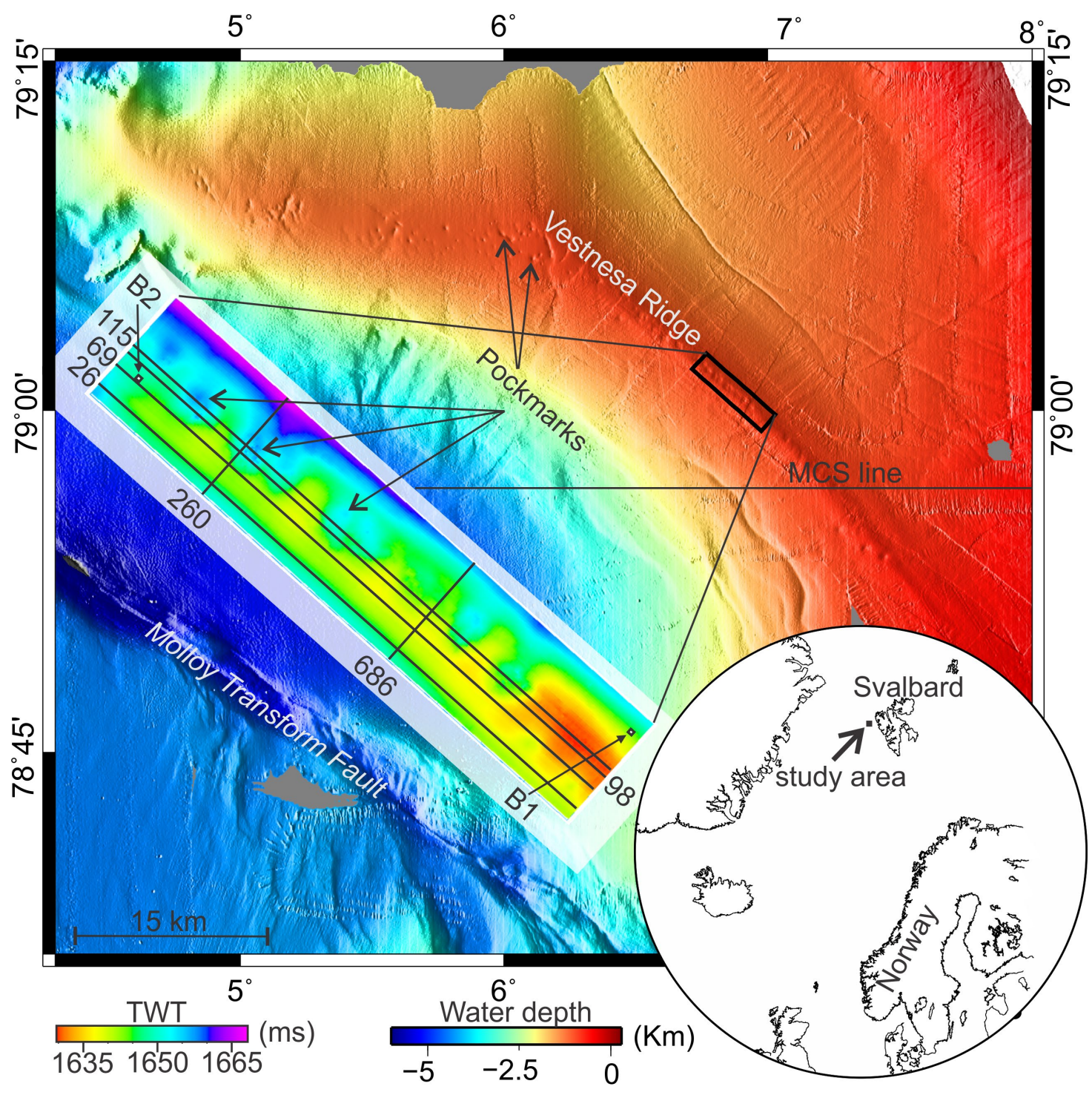


Figure 2
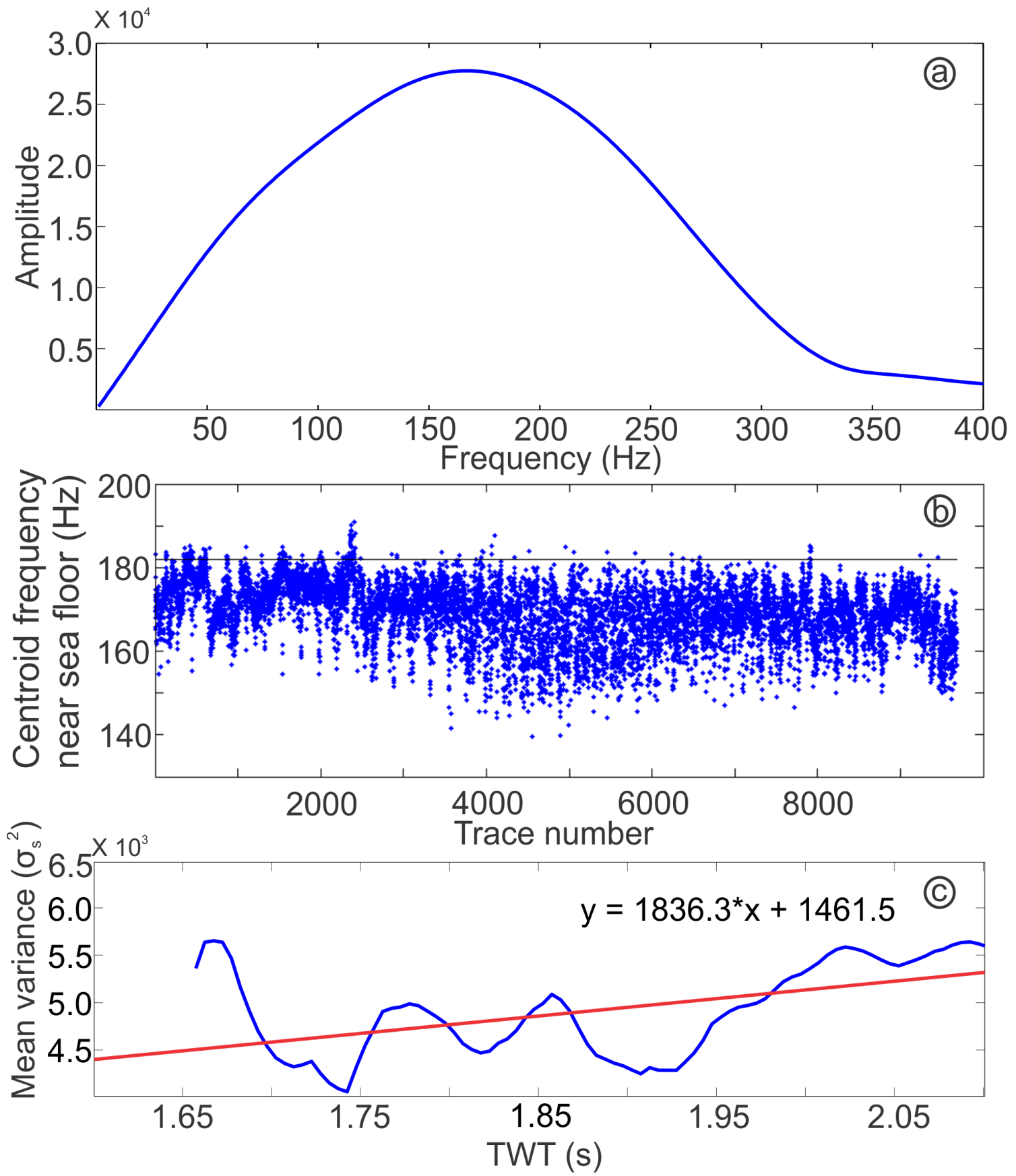
Figure 3
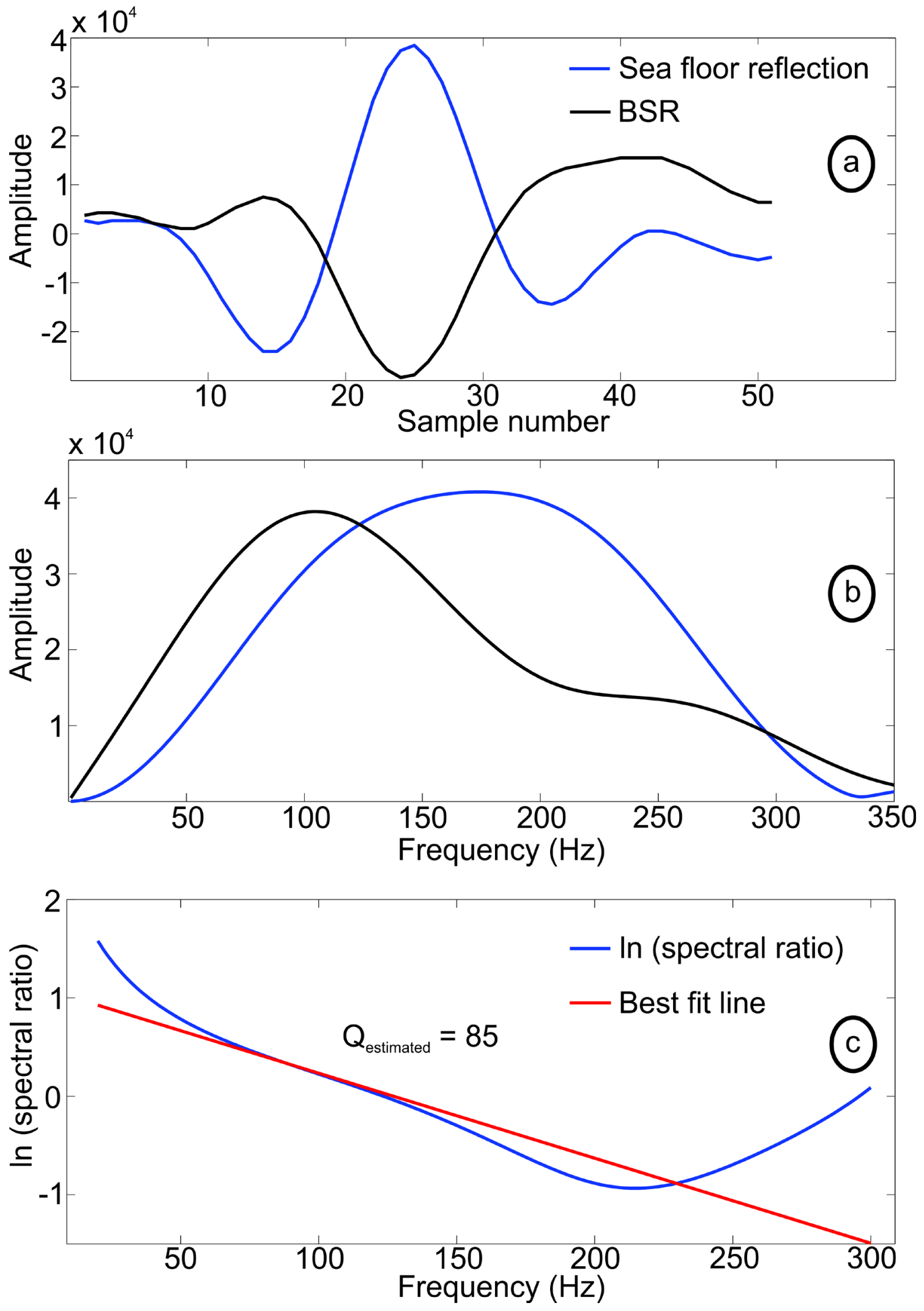
Figure 4
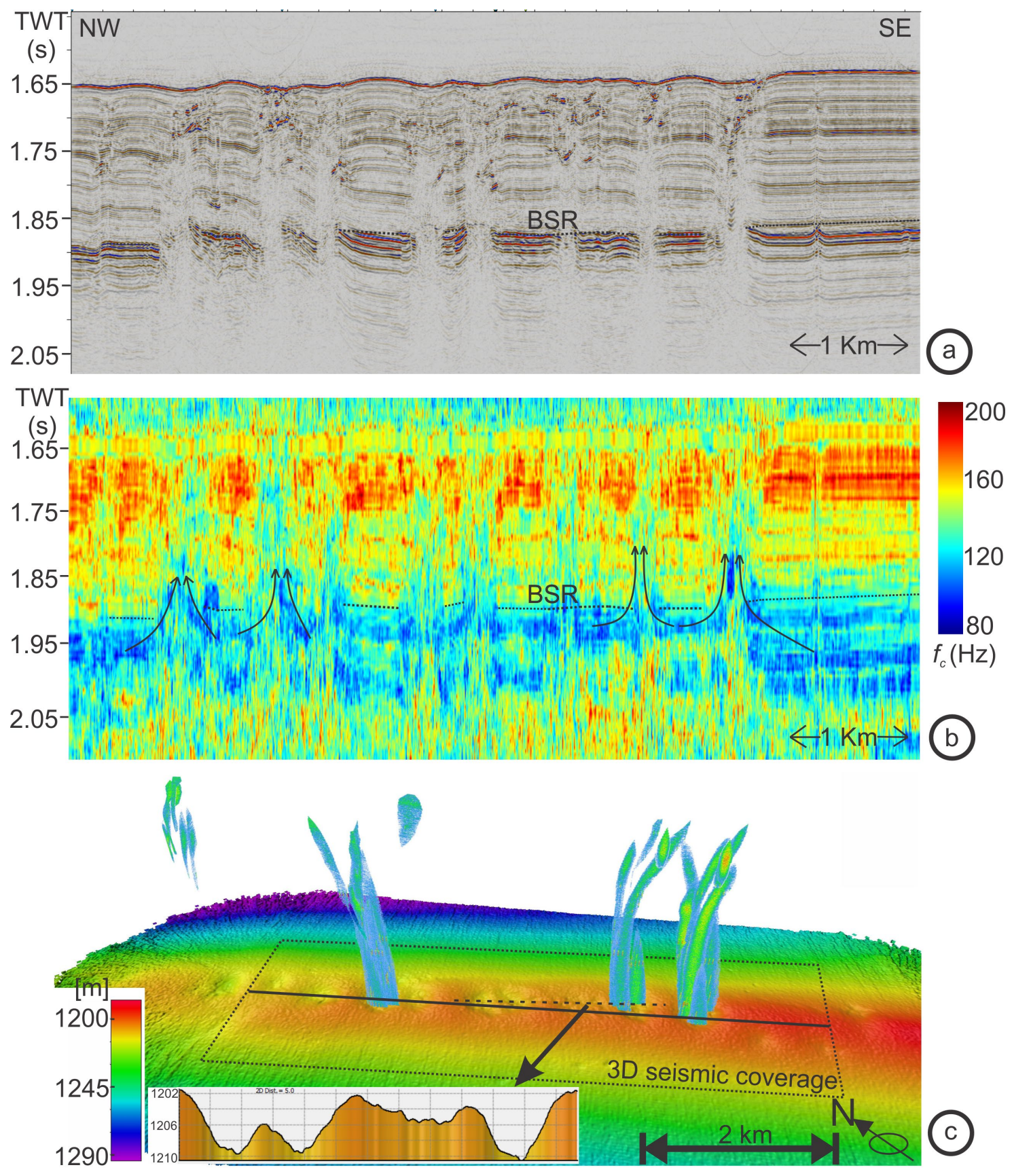
Figure 5

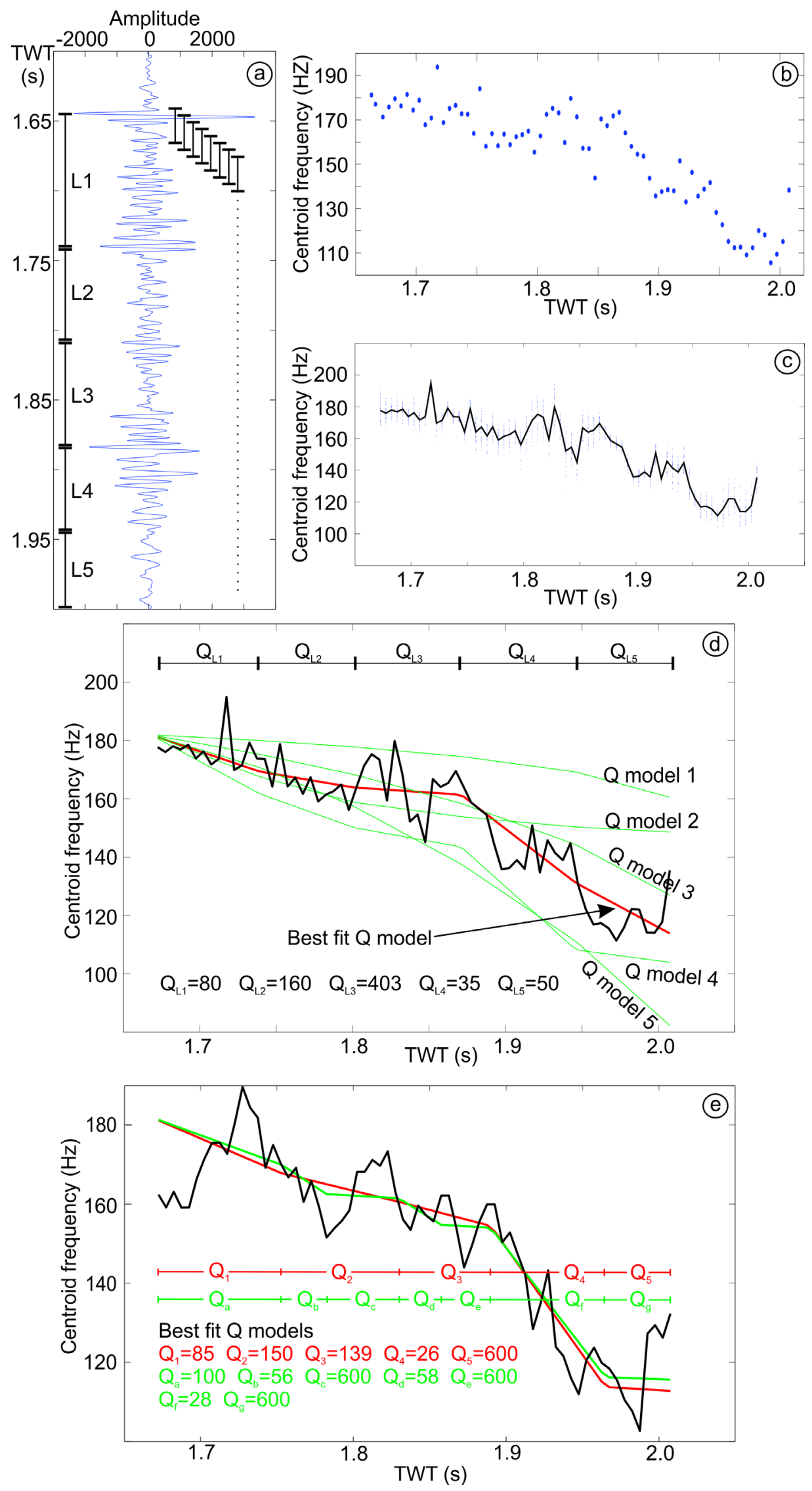


Figure 6
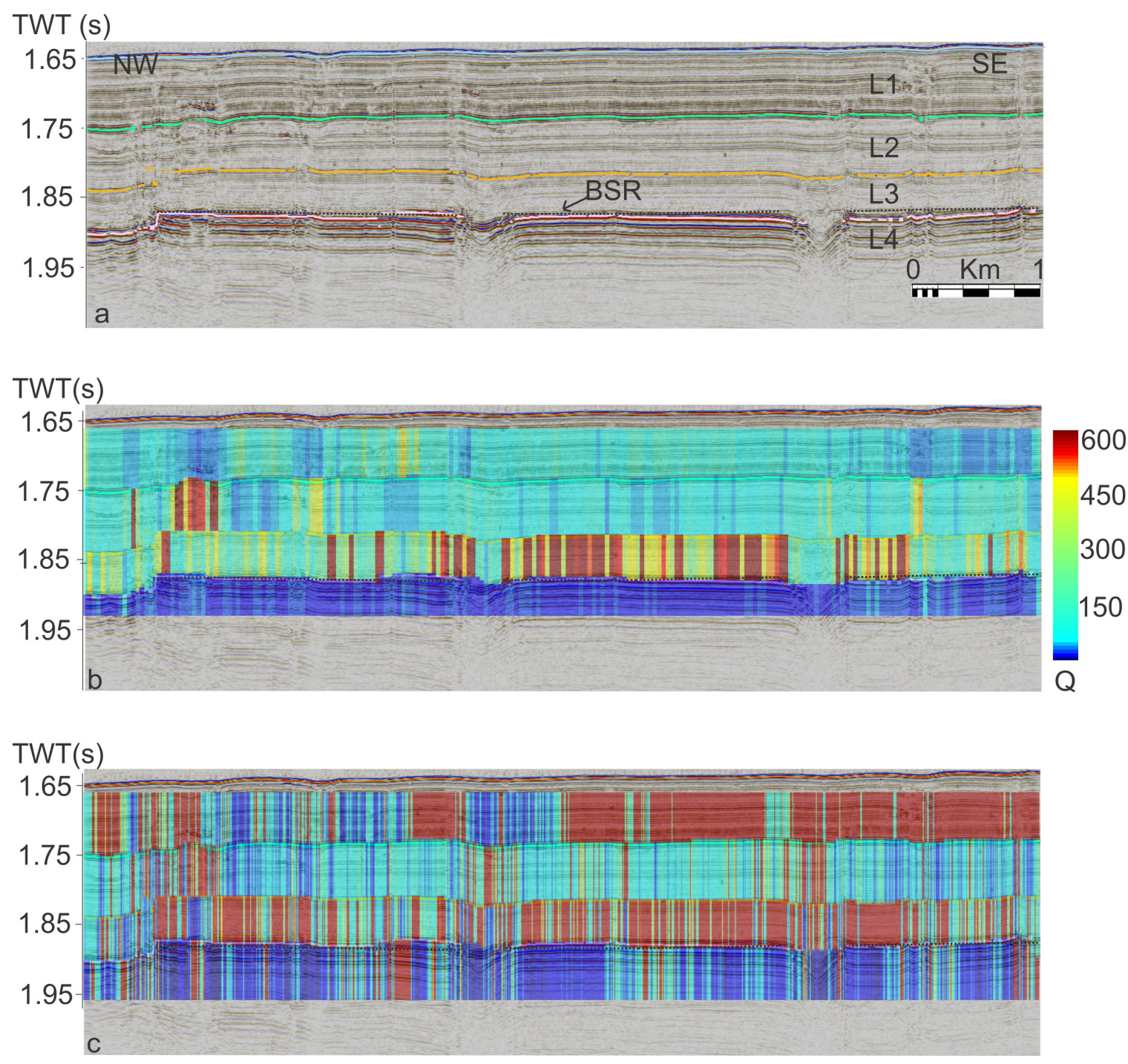
Figure 7

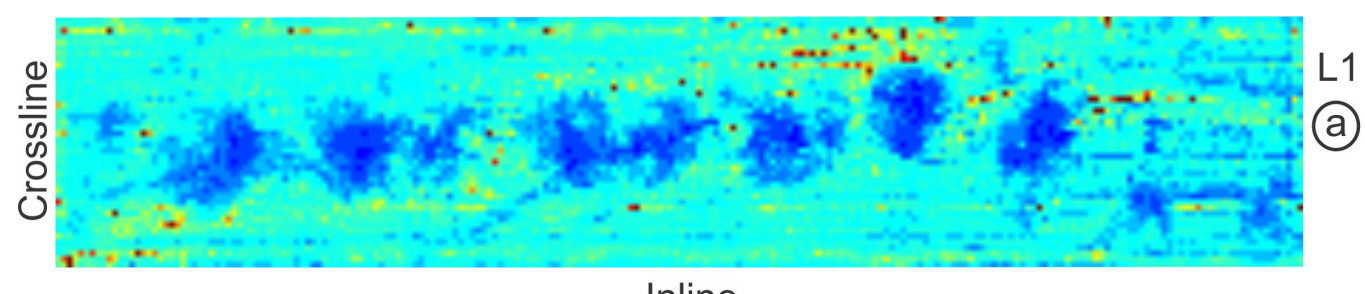

Inline

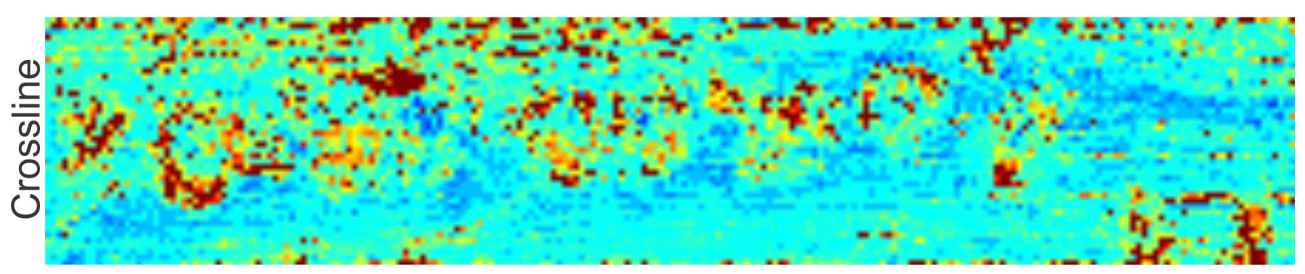

Inline
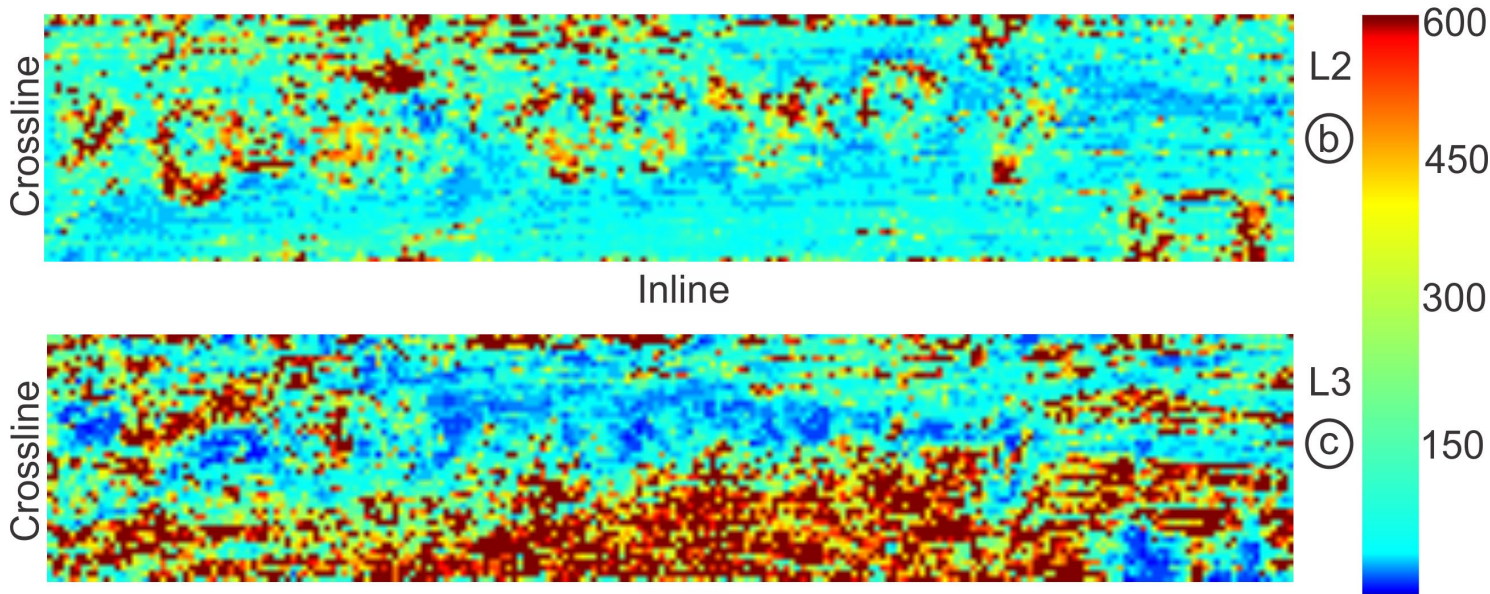

Inline $----\mathrm{BSR}----$
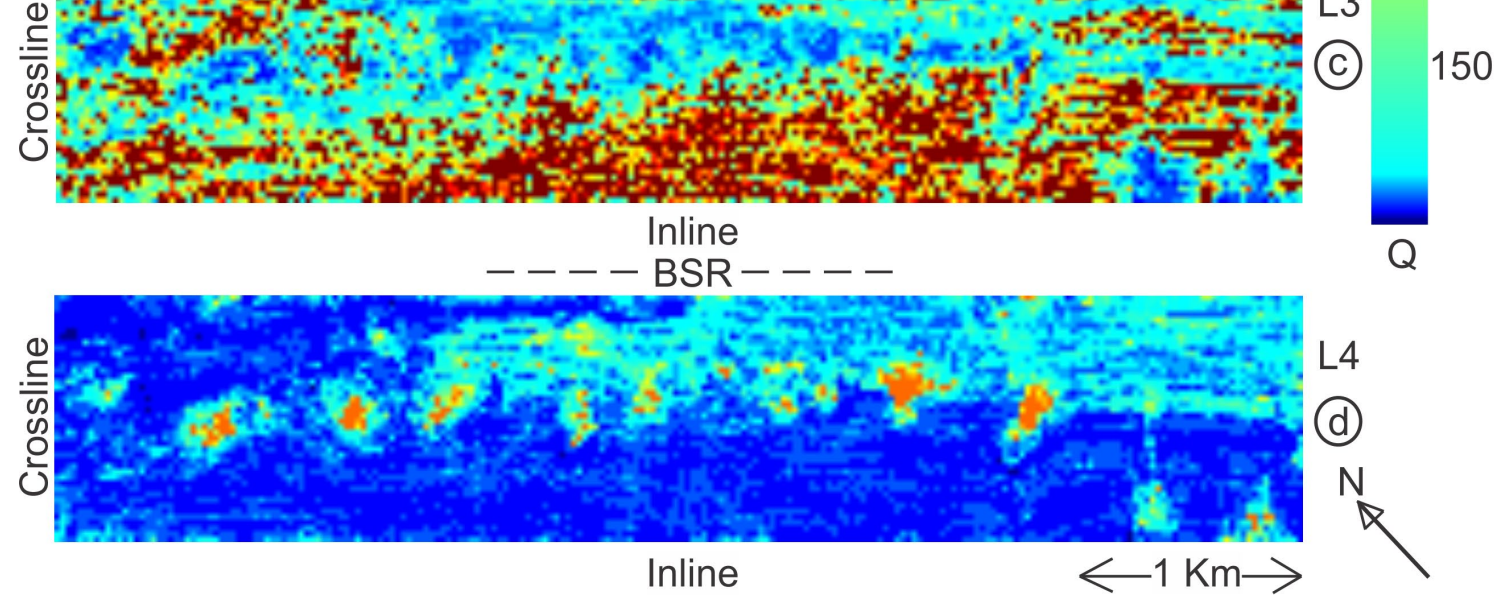
Figure 8
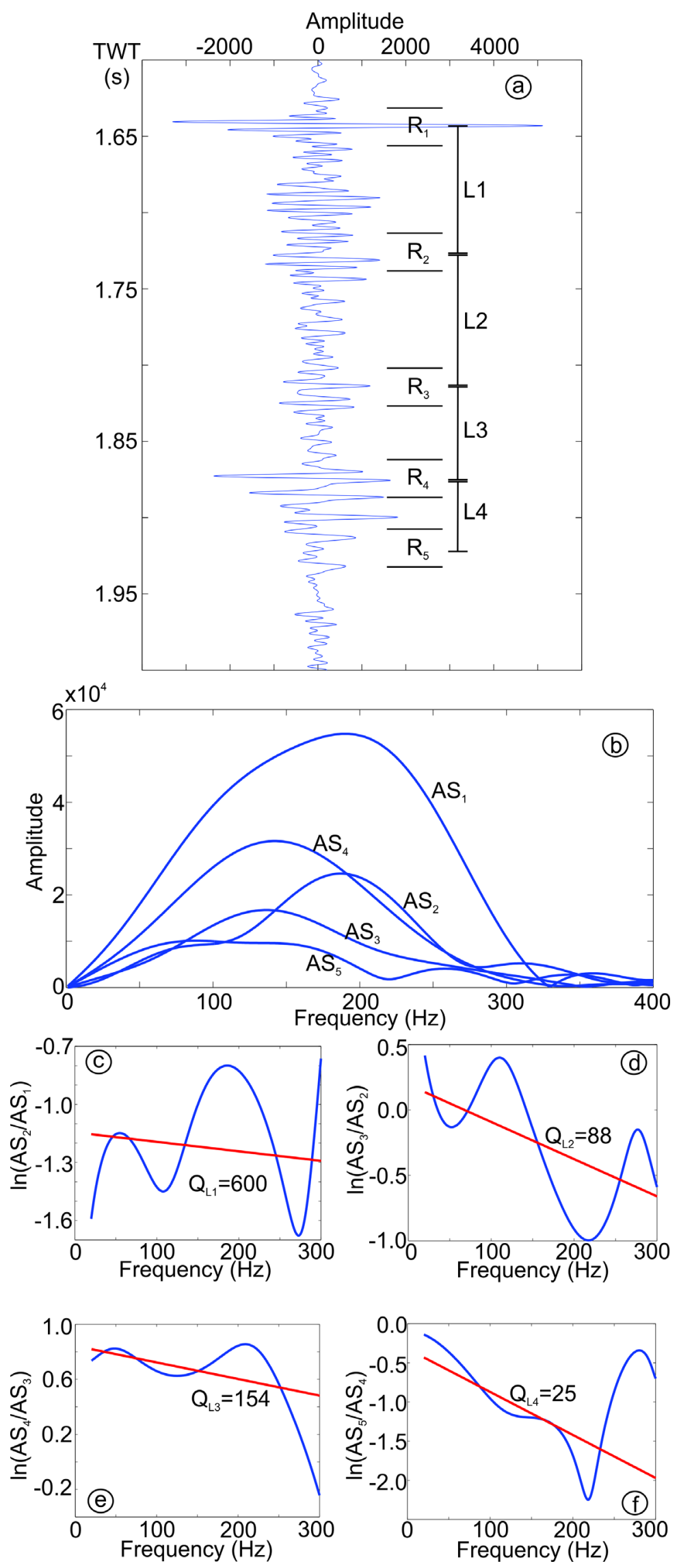
Figure 9
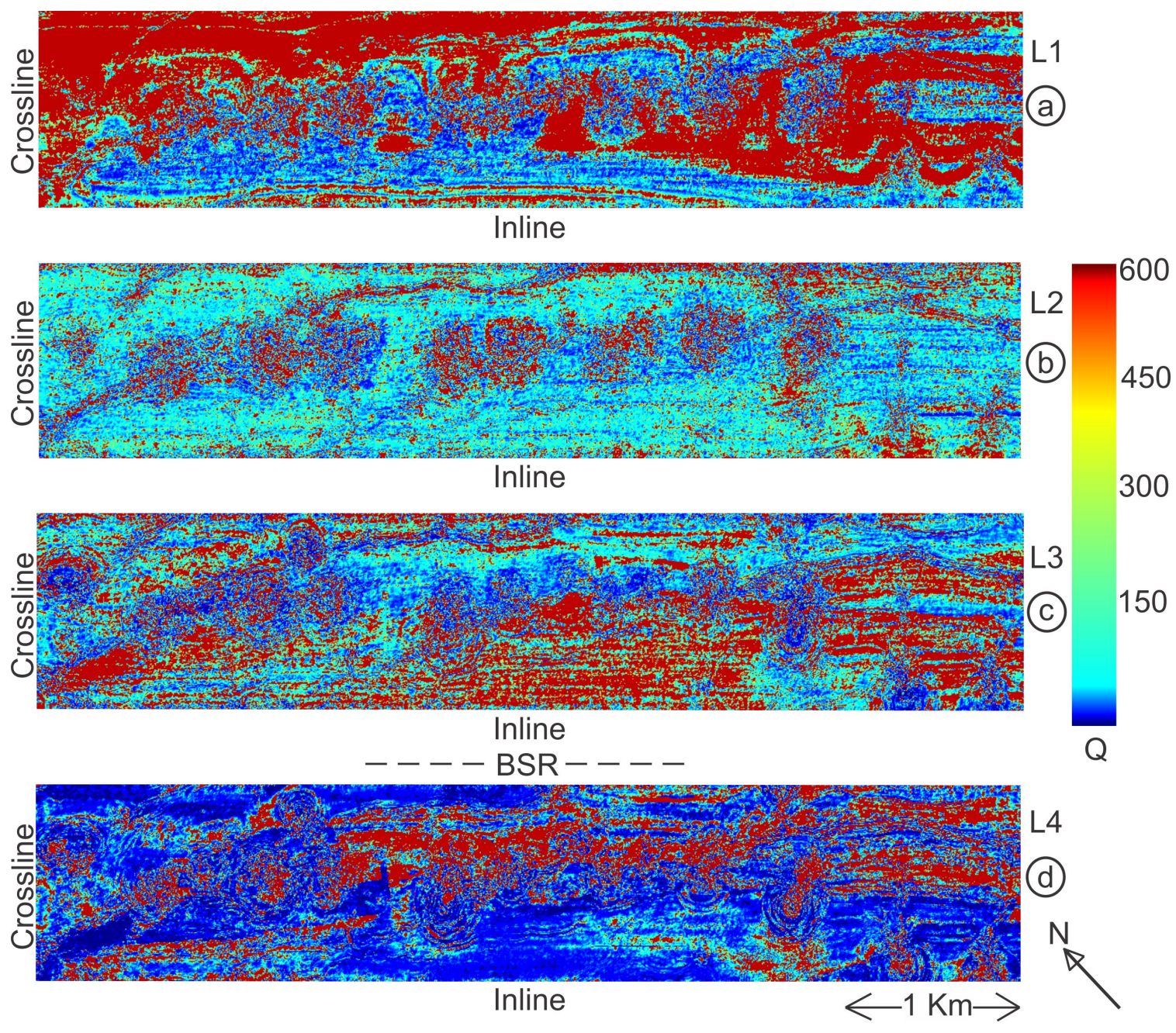

L4

(d)

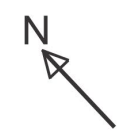


Figure 10
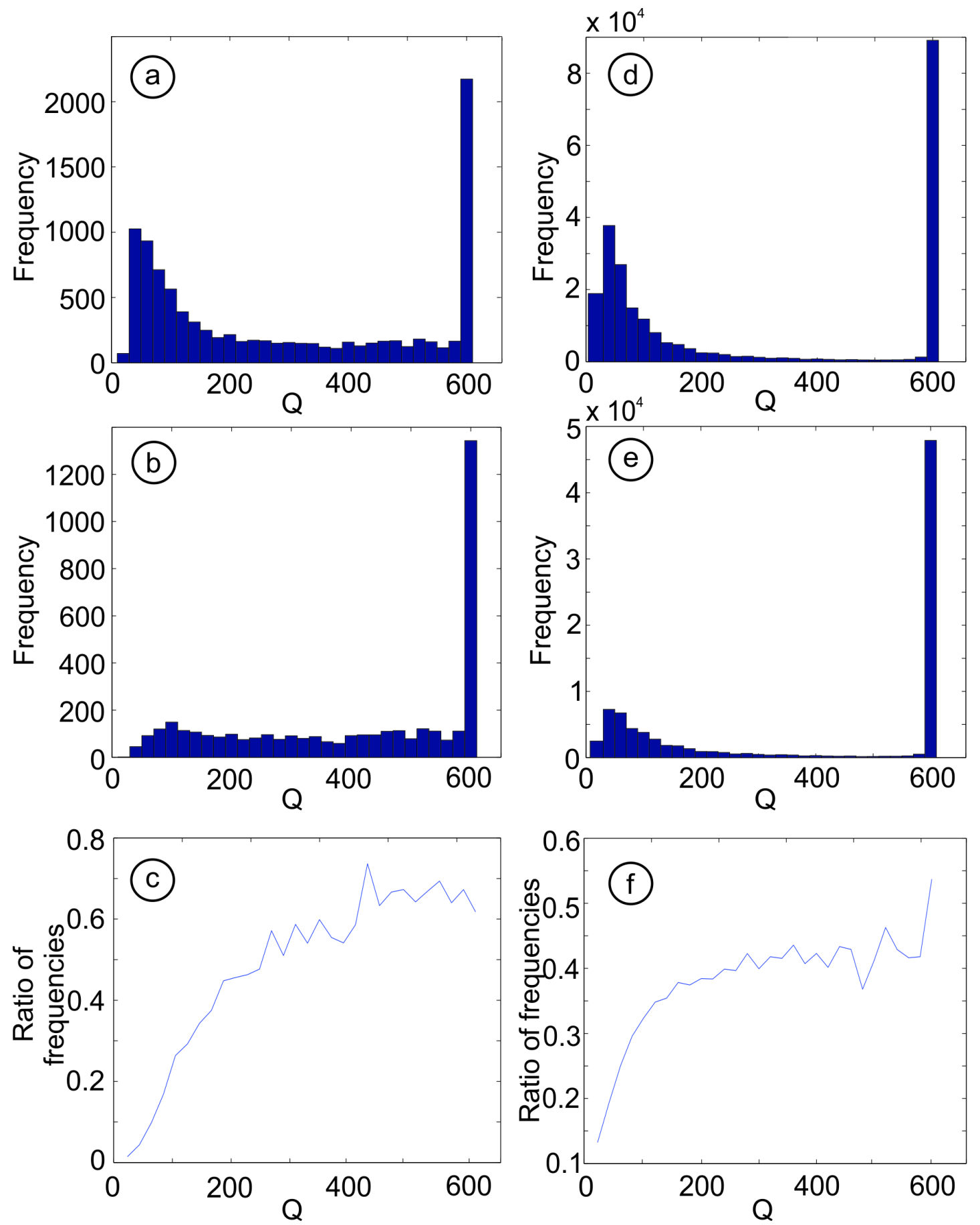
Figure 11
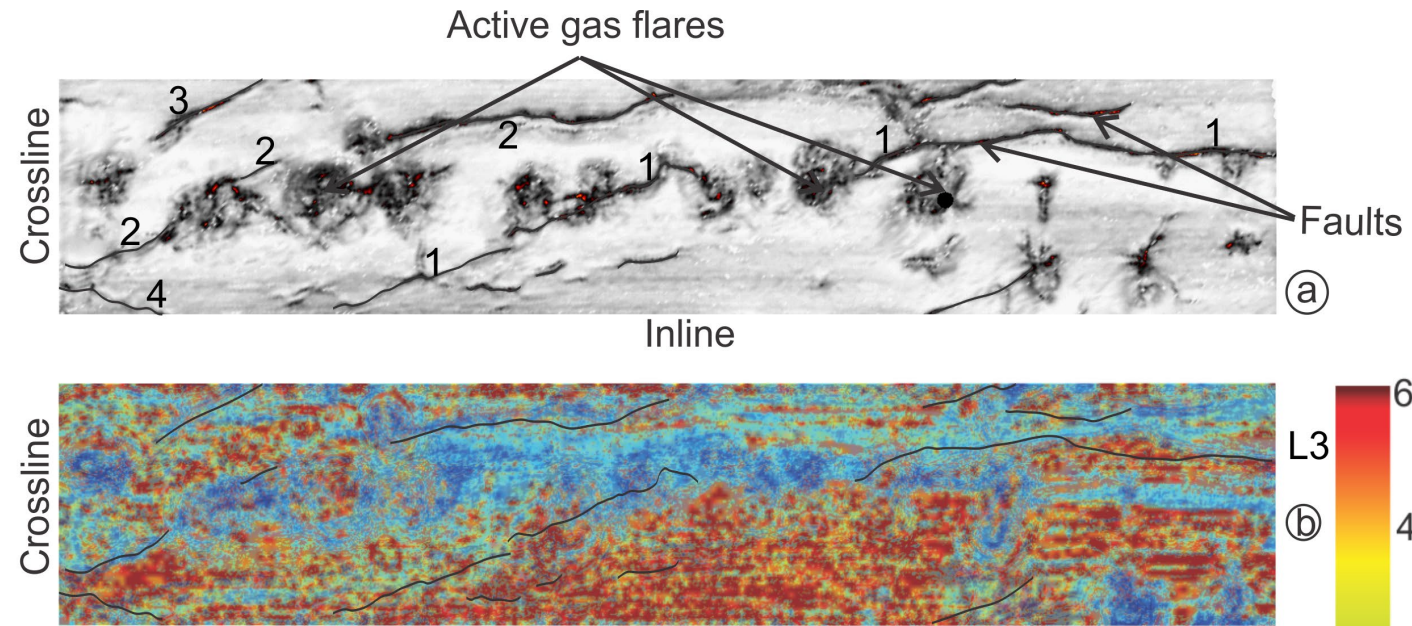

Inline
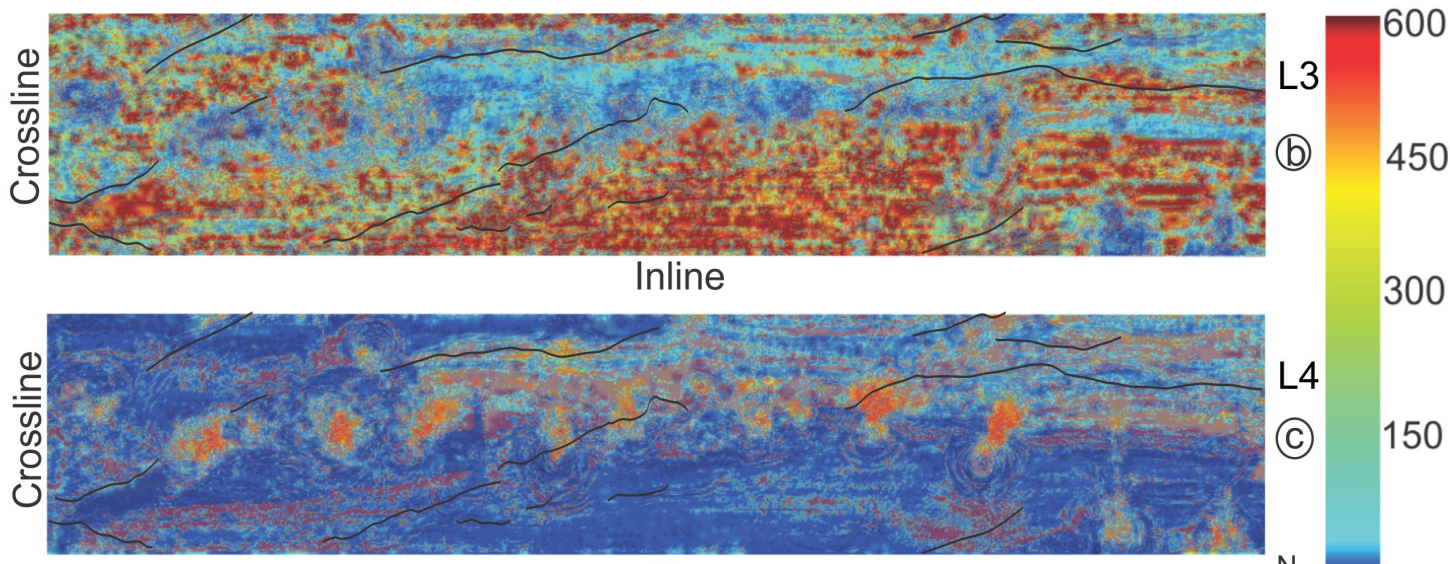

Inline

L4

(C) 150

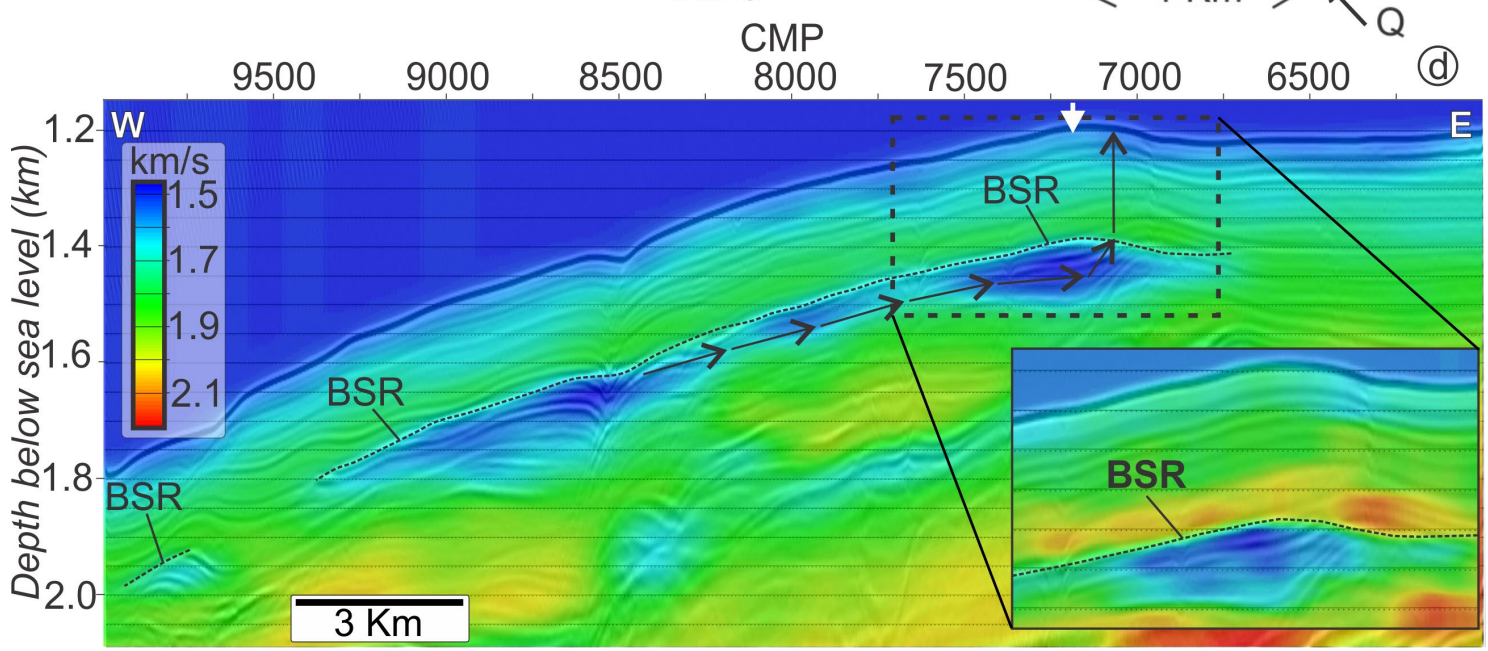

